\title{
Characterisation of Titanium Dental Implants I: Critical Assessment of Surface Roughness Parameters
}

\author{
Johanna Löberg $^{1,2}$, Ingela Mattisson ${ }^{2}$, Stig Hansson ${ }^{2}$ and Elisabet Ahlberg ${ }^{*}{ }^{1}$ \\ ${ }^{I}$ Department of Chemistry, University of Gothenburg, SE-41296 Gothenburg, Sweden \\ ${ }^{2}$ Astra Tech AB, SE-431 21 Mölndal, Sweden
}

\begin{abstract}
Titanium is commonly used for dental implants because of its unique ability to get incorporated into living bone. There is an ongoing development to obtain better anchorage and surface properties such as roughness and chemical composition are modified to reach this. In this study titanium dental implant surfaces were characterised by recording the topographical changes induced by each individual processing step such as cleaning, blasting, and HF etching. To fully describe the different surfaces, the same point was analysed before and after each step using Atomic Force Microscopy (AFM) and 3D-Scanning Electron Microscopy (3D-SEM). A set of 3D surface parameters were calculated as a function of filter size to describe the topographic features at different levels. The chemical treatment introduces nano-sized features while blasting changes the topography at the micrometer level and by combining AFM and 3D-SEM the entire range can be assessed. The results show that the chemically induced changes in the topography can only be revealed by AFM while 3D-SEM gives a clear description of the topography of blasted surfaces. The fractal dimension for the chemically treated surface was the same as for the blasted surfaces but crossover size was much smaller. Besides the commonly used $S_{a}$ parameter it is suggested that the root-mean-square of the surface slope $\left(S_{\mathrm{dq}}\right)$ and the void volume $\left(\mathrm{V}_{\mathrm{vc}}\right)$ parameters are included in the characterisation of rough surfaces. These parameters can be used for correlation with in vivo performance.
\end{abstract}

Keywords: Surface roughness parameters, 3D-SEM, AFM, titanium dental implants, fractal analysis.

\section{INTRODUCTION}

It has been shown that the topography and surface composition of dental implants affect the interaction with the surrounding bone tissue [1-6]. To induce the desired biological response, different surface modifications are used, for example anodic oxidation, blasting, ion-incorporation, coating with bioactive substances, heat treatment etc. [7,8]. When surface modifications affecting the macro- and microscale topography are used changes on the sub-micro topography are often induced [9]. It has been found that roughened implant surfaces give increased retention strength in vivo [9-16] and that topographies down to the nanometre range affect the attachment and growth of bone cells [17, 18]. This makes topographical characterisation important. A dental implant often exhibits topographical features ranging from millimetre (threads) down to sub-micro and/or nanometre scale. Today, no single technique is able to analyse this whole range and complementary techniques [13, 19-23] as well as combinations of different techniques have to be used $[20,21]$. The appropriate technique to be used depends on the specific question asked, for example the size range of the surface that needs to be analysed and by the instrumental limitations such as measuring length or area and the vertical resolution [24].

The topography of a surface can be divided into three different categories: form, waviness and roughness depending on the wavelength or peak-to-peak spacing of the

*Address correspondence to this author at the Department of Chemistry, University of Gothenburg, SE-41296 Gothenburg, Sweden; Tel: +46 31 7869002; Fax: +46 31 7722853; E-mail: ela@chem.gu.se surface features [25]. Conventionally, the waviness and form are removed by filtering and numerical surface parameters are often calculated on the roughness alone and are thereafter called surface roughness parameters [25]. The surface roughness parameters either describe surface characteristics in two dimensions, 2D (marked by $\mathrm{R}$ ), or in three dimensions, 3D (marked by S). ISO standards for how to characterise surfaces in 2D exist [26] while an ISO standard for 3D characterisation is under development [27]. In 2D surface characterisation a number of profiles of the surface are evaluated while in 3D areas are analysed, which gives a more comprehensive description of the surface topography. With the development of new characterisation techniques, analysis in 3D is more common. 3D surface roughness parameters are divided into different groups depending on what surface characteristics they are describing: 1) amplitude, 2) spatial, 3) hybrid, 4) volume and area, and 5) functional parameters [28-30]. Amplitude parameters describe the amplitude property of the topographical features which are considered to be the most important property of the surface [29]. The spatial parameters describe the texture, randomness, and periodicity of the surface while the hybrid parameters are combinations of spatial and amplitude parameters where a change in either of the two properties affects the value of the hybrid parameter. The volume and area parameters are defined from the bearing curve of the surface and describe the bearing and void property of the topography. Functional parameters are more specific parameters which describe particular characteristics of a surface such as fluid retention and bearing [28, 30, 31].

Surface roughness parameters are used to describe dental implant surfaces. However, a wide spread can be seen in 
surface parameter values published by different groups for nominally the same surfaces [32, 33]. MacDonald et al. [33] had 7 specimens of different surface textures analysed both by similar and different techniques at three international laboratories. The results showed a variation of $\pm 300-1000 \%$ depending on the techniques used and the type of surface. The large spread in obtained values was discussed in terms of usage of inappropriate characterisation techniques, inaccurate or missing information regarding filter size and type of filter, as well as differences in instrumental settings and how different surface topography levels were characterised [32, 33]. Thus, when comparing surface roughness parameters obtained from different instruments, it is important to state the settings and spatial resolution [22, 23], otherwise the obtained parameter values may describe different levels on the surface. To overcome the above problems, Wennerberg et al. [32] published a guideline for characterisation of dental implant surfaces, giving recommendations about analysing procedure, preferred techniques, relevant surface roughness parameters together with information regarding data filtering. It was recommended that at least one amplitude, one spatial and one hybrid parameter should be used. Other ways of characterising dental implant surfaces have been published $[20,31,34]$ including the use of Fast-Fourier-Transform (FFT) methods where a wavelength dependence of the roughness is obtained [22, 23]. The relevance in using surface roughness parameters to describe dental implant surfaces has been questioned since surfaces with very different appearance have similar parameter values $[35,36]$.

The aim of the present study was to critically assess the value of surface roughness parameters in discriminating between different surfaces. To accomplish this, the same part of the surfaces studied was analysed sequentially after each processing step. Thirteen 3D surface roughness parameters were thoroughly evaluated using two different characterisation techniques with different spatial resolution, Atomic Force Microscopy (AFM) and 3D-Scanning Electron Microscopy (3D-SEM). By using these two characterisation techniques and applying Gaussian filters of different cutoffs, changes in topography can be measured from low millimetre down to nanometre level. The outline of the paper is the following. In section 2 the surface pre-treatment is described and in section 3 the surface roughness parameters are defined and a qualification of the method used to extract the parameters is given. In section 4 the results are given followed by a discussion in section 5 of the feasibility of using the obtained parameters for discrimination between different pre-treatment steps. In a forthcoming paper, calculated interface shear strengths for the same surfaces will be reported and discussed in the context of the surface characterisation presented in the present paper [37].

\section{METHOD}

\subsection{Samples}

Commercially pure titanium discs (Grade IV) with turned surface were used as initial material. The samples (diameter $6.25 \mathrm{~mm}$ ) were marked with a milled cross creating four quadrants with one of the quadrants marked for visual separation. This made it possible to perform topographical measurements on the same spot after sequential processing steps. In total, three different samples were used in this study where five different surfaces were analysed (Fig. 1). $i$ ) turned surface (TS), ii) TS surface treated with diluted hydrofluoric acid (TS+HF), iii) TS blasted with small $\mathrm{TiO}_{2}$ particles, i.e. fine blasted surface (FB), representing the surface of the previously commercially available TiOblast $^{\mathrm{TM}}$ implant (AstraTech $\mathrm{AB}$ ), iv) TS surface blasted with large $\mathrm{TiO}_{2}$ particles, i.e. coarse blasted surface (CB), v) CB surface treated with diluted $\mathrm{HF}(\mathrm{CB}+\mathrm{HF})$, representing the surface of the commercially available dental implant OsseoSpeed ${ }^{\mathrm{TM}}$ (AstraTech AB).

The TS and CB surfaces were used as references for the $\mathrm{TS}+\mathrm{HF}$ and $\mathrm{CB}+\mathrm{HF}$ surfaces, respectively. The HF treatment was performed according to the OsseoSpeed ${ }^{\mathrm{TM}}$ process which introduces a smaller topographic structure on top of the underlying surface (Fig. 1). The samples were created and analysed according to the scheme shown in Fig. (2), where the TS surface was first analysed and then transformed into the TS+HF surface by the HF treatment. In the same way the $\mathrm{CB}$ surface was first analysed and transformed into the $\mathrm{CB}+\mathrm{HF}$ surface. The influence of the cleaning processes on the topography was investigated and no changes in the surface parameters could be seen. Three points per surface were analysed after each treatment. To follow the topographical changes, area analysis was performed on approximately the same spot after each treatment. On the turned samples (TS, TS+HF), finding the same point was straight forward but more difficult on the blasted surfaces. However, approximately the same points were analysed also on the blasted samples. By performing topographical measurements at the same point after each processing step, detailed information on how each step influences the topography was obtained.

\subsection{Topographical Analysis Techniques}

To measure both topography from underlying structure together with the topography introduced by the HFtreatment, two different surface sensitive techniques were used; Atomic Force Microscopy (AFM) and 3D-Scanning Electron Microscopy (3D-SEM). The data obtained were imported into the calculation software $\mathrm{MeX}^{\circledR}$ [38], where 3D-roughness parameters were calculated (further discussed in section 3). The $\mathrm{MeX}^{\circledR}$ software is designed to calculate surface roughness parameters from SEM images but was utilised in this work also for analysing AFM data to avoid any differences depending on the software used.

\subsection{AFM}

AFM measurements were performed on a Nanoscope ${ }^{\circledR}$ IIIa (Digital Instruments) equipped with Nanoscope ${ }^{\mathbb{B}} 5.12$ software, using Tapping Mode. AFM measurements were only possible on the TS and TS+HF samples because of limitations in maximum vertical resolution (maximum limit $5 \mu \mathrm{m}$ ). The probe model used was RFESP7 from Veeco Instruments Inc. All analysed areas were recorded with three different scan sizes, $10 \times 10,5 \times 5$ and $3 \times 3 \mu \mathrm{m}$. A scan frequency of $0.8 \mathrm{~Hz}$ and 256 scan lines were used for TS surface while 512 scan lines were used for TS+HF surface.

\subsection{D-SEM}

SEM images were collected using an ESEM XL30 (FEI Company), software XL Microscope Control 7.00, with an acceleration potential of $30 \mathrm{kV}$ and secondary electron (SE) 


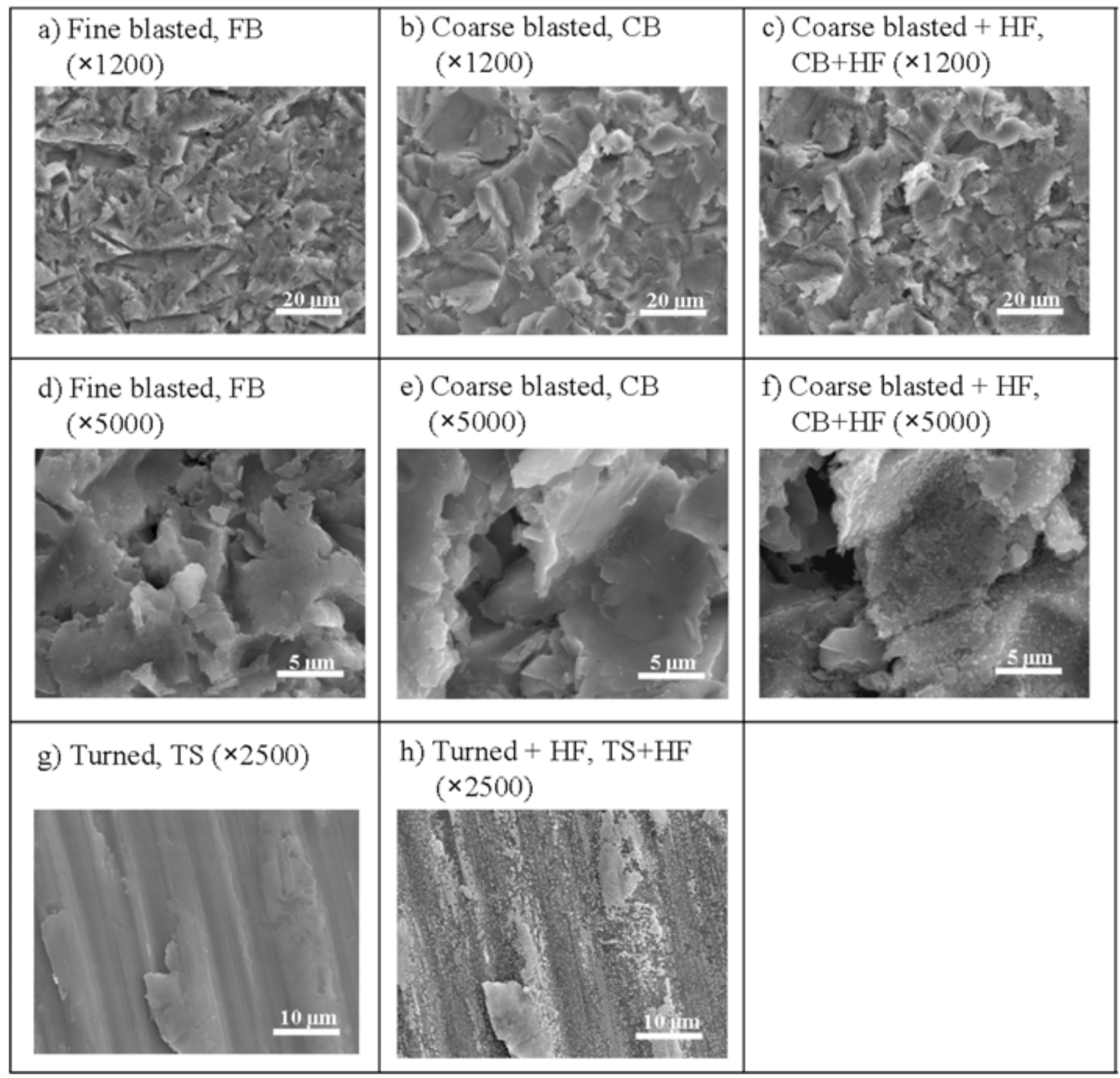

Fig. (1). SEM images of the different surfaces. (a-c) show the blasted surfaces at $\times 1200$ magnification while (d-f) show the same surfaces at $\times 5000$ magnification. $\mathbf{g}$ ) and $\mathbf{h}$ ) show the turned and etched surfaces at $\times 2500$ magnification.

detector. Spot sizes used on the different surfaces were; TS) 5.2, TS+HF) 4.8, FB) 4.8, CB) 4.6, CB+HF) 4.2. The working distance, which is the distance between sample and detector, varied between 9.5 to $10.0 \mathrm{~mm}$. To induce 3Dvisualisation with the SEM technique, stereo-pairs were collected by tilting the sample around the same point on the surface with a tilting angle of $5.6^{\circ}$ and $11.2^{\circ}$ for the blasted and turned surfaces, respectively. Images were collected with similar contrast and brightness settings to prevent differences in parameter values when analysed by the $\mathrm{MeX}^{\circledR}$ software.

By using the microscope program, SEM images were collected in XHD (Extra High Definition) format which contains more information and have more pixels than regular
TIF images (images collected at $\times 500$ magnification with TIF and XHD have a resolution of $384.4 \mathrm{~nm} /$ pixel and 96.25 $\mathrm{nm} /$ pixel, respectively). Stereo pairs were collected at three or four different magnifications: $\mathrm{CB}$ and $\mathrm{CB}+\mathrm{HF}$ samples, $247.84 \times 186.24 \mu \mathrm{m}(\times 500), 103.26 \times 77.594 \mu \mathrm{m}(\times 1200)$ and $24.784 \times 18.624 \mu \mathrm{m}(\times 5000)$. For the $\mathrm{TS}+\mathrm{HF}$ and $\mathrm{FB}$ surfaces, an additional surface magnification was analysed to follow the small changes induced, $49.569 \times 37.249 \mu \mathrm{m}$ $(\times 2500)$. Blurry images were received at $\times 5000$ magnification for the TS surface and the surface was analysed at $\times 500, \times 1200$, and $\times 2500$ magnification. By collecting stereo-images at different magnification, overlaps in the analysed areas were created. The same three points analysed in the AFM analysis, were also analysed by the 3D- 
Turned
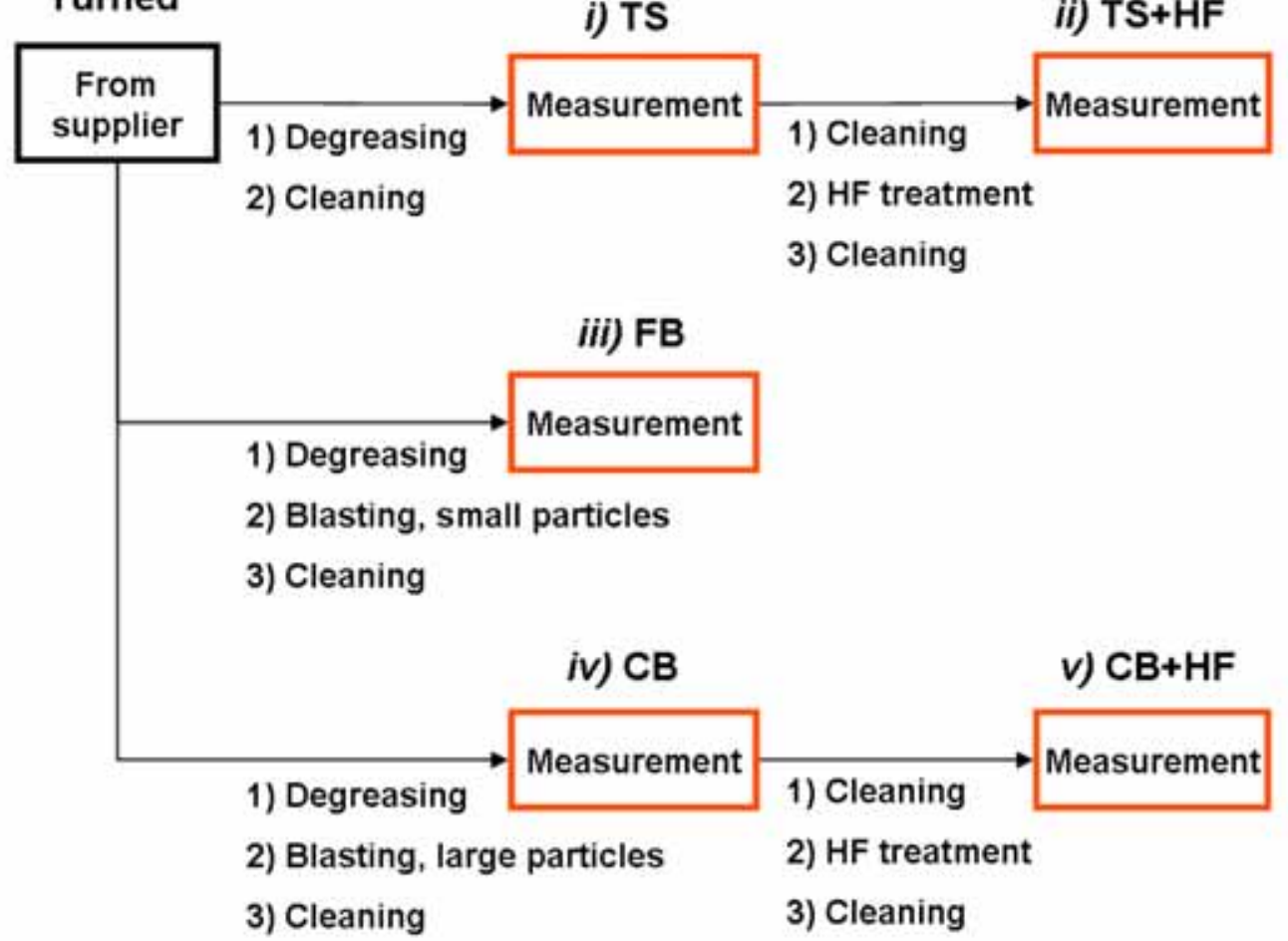

Fig. (2). Scheme over the preparation and measurement process.

SEM technique, creating an overlap between the two techniques.

\section{DATA ANALYSIS WITH THE MEX ${ }^{\circledR}$ SOFTWARE [38]}

To transfer stereo-SEM images into a matrix of numbers and to calculate 3D-surface roughness parameters, the $\mathrm{MeX}^{\circledR}$ software from Alicona Imaging [38] was used. The stereopair images were imported together with information regarding the total titling angle, pixel size of the images and the working distance in the microscope. From this information 3D-models were created where different greylevels correspond to different heights [39]. The software allows the possibility to create 3D-models from two (left and right) or three (left, right, and middle) SEM images. Two images were used in this study since no difference in parameter values were received when using two or three images. AFM ASCII files were imported into the $\mathrm{MeX}^{\circledR}$ software and all AFM and 3D-SEM data were analysed according to the method described below.

Both 2D and 3D-surface roughness parameters are used in the literature for describing dental implant surfaces. However, since surfaces interact in three dimensions, 3Dsurface roughness parameters have a clear advantage over 2D parameters for describing the real situation and the area analysis mode available in the $\mathrm{MeX}^{\circledR}$ software was used to calculate 3D-roughness parameters. Parameters can be calculated for the original image (in the $\mathrm{MeX}^{\circledR}$ software called primary parameters) as well as after the application of filters. The filtering function available in the $\mathrm{MeX}^{\circledR}$ software applies a Gaussian filter with a bandwidth of 100. In the present study, a roughness filter with five different cut-offs were applied, defined as $20,15,10,5$ or $1 \%$ of the horizontal width of the image. By applying a roughness filter, wavelengths of larger sizes than the cut-off are removed. Filtering together with different SEM magnifications and AFM scan sizes made it possible to retrieve topographical information down to a few hundred nanometres.

To qualify the method of analysing the AFM and 3DSEM data used in this work, different options within the $\mathrm{MeX}^{\circledR}$ software were evaluated. Tests regarding the placement of the reference plane, contrast settings when collecting the SEM images and filtering options, were made. One of the most important settings when analysing with the $\mathrm{MeX}^{\circledR}$ software is the location of the reference plane of the image, since many of the mathematical surface roughness parameters are defined in relation to this reference plane. An incorrectly placed reference plane will lead to erroneous parameter values. The test comprised manual versus automatic adjustment of the reference plane and deliberately tilting the sample before automatic generation of the reference plane. For each setting the surface parameter values were evaluated. The results showed that the Robust Adjustment function available in the $\mathrm{MeX}^{\circledR}$ software was preferable since it yielded the highest reproducibility. By the Robust Adjustment, the reference plane is fitted onto the most even part of the image, and is not influenced by peaks and valleys. A new reference plane was calculated for each magnification and filter size. In the $\mathrm{MeX}^{\circledR}$ software the 3DSEM image is converted to a $3 \mathrm{D}$ matrix of numbers. It is therefore important that the brightness and contrast are adjusted to get images showing most of the topographical features present on the surface. The resolution is not very sensitive to the contrast and brightness settings provided that extreme values are avoided, i.e. too light or dark images. In 
the following analysis, SEM images were collected with similar settings in contrast and brightness.

\subsection{D-Surface Roughness Parameters}

A set of 3D-parameters for detailed description of all kinds of engineering surfaces has been suggested by Dong, Sullivan and Stout [28-30]. The set includes height, spatial, hybrid, functional, volume and area 3D parameters. Many of the parameters suggested by these authors and a few more are accessible in the $\mathrm{MeX}^{\circledR}$ software. All these parameters were evaluated with respect to the usefulness in characterising dental implant surfaces. A short list of parameters were chosen and complemented with parameters commonly used in literature for characterisation of dental implant surfaces. The chosen parameters are shown in Table $\mathbf{1}$ and are separately discussed below.

\subsubsection{Amplitude Parameters}

The amplitude is considered to be the most important property of a surface topography [29]. The amplitude parameters can be divided into three categories after the properties they are describing, $(i)$ statistical characteristics of surface height; (ii) extreme characteristics of surface height; and (iii) the shape of surface height distributions. The average height parameter, $\mathrm{S}_{\mathrm{a}}$, is the parameter most frequently used for describing dental implant surfaces. In addition to the $S_{a}$ parameter, the statistically more significant $S_{\mathrm{q}}$ parameter (root-mean-square of the average height) [29] was chosen for evaluation. The $\mathrm{S}_{\mathrm{a}}$ and $\mathrm{S}_{\mathrm{q}}$ parameters are strongly coupled and are sensitive to the size of the sampling area but insensitive to the sampling interval [30]. To receive information regarding the absolute height of the surface, the $\mathrm{S}_{10 \mathrm{z}}$ parameter was chosen. This parameter gives the average value of the absolute height of the five highest peaks and

Table 1. Surface Area Parameters Chosen in this Study

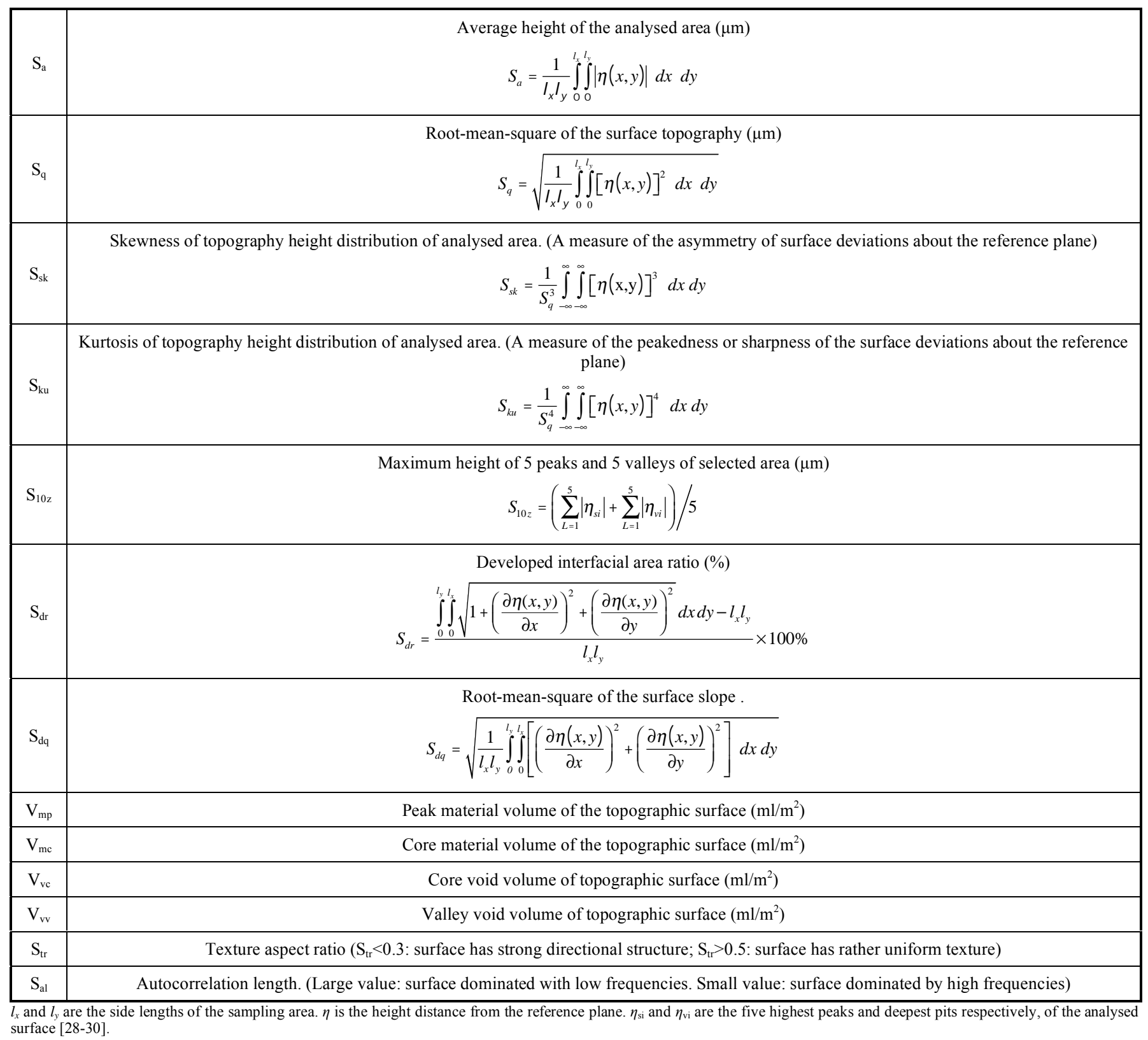




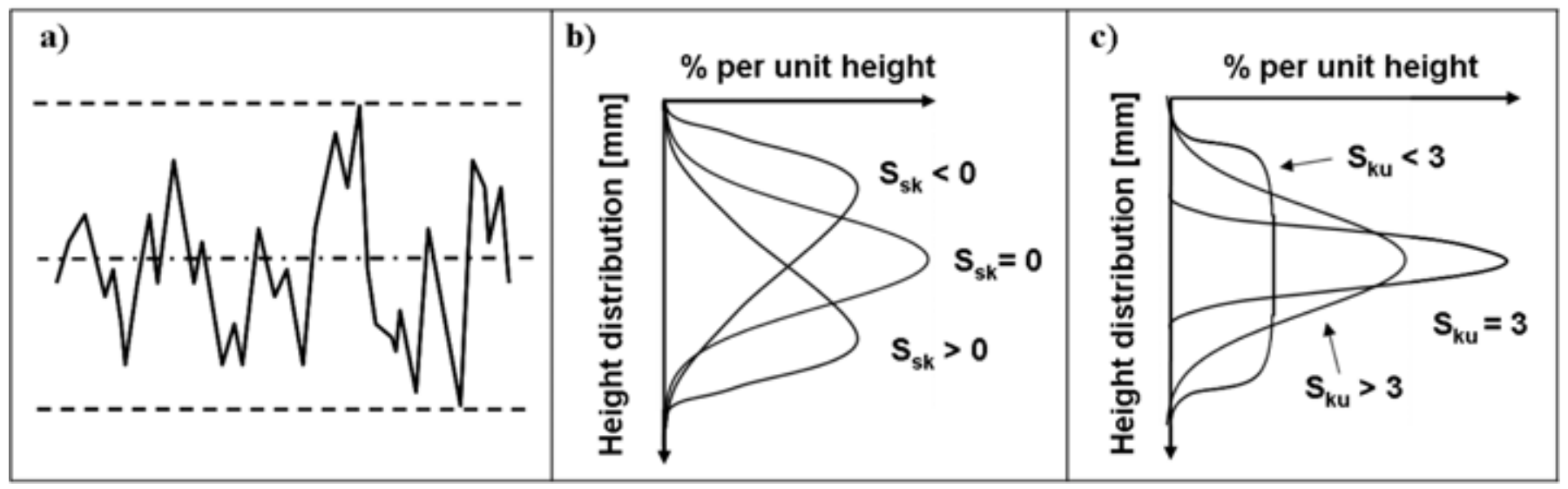

Fig. (3). The amplitude parameters skewness $\left(S_{\mathrm{sk}}\right)$ and kurtosis $\left(S_{\mathrm{ku}}\right)$ are defined from the amplitude distribution curve. (a) Shows the surface simplified to a profile and (b) and (c) show examples of different skewness and kurtosis values. For a Gaussian surface $S_{\text {sk }}=0$ and $S_{k u}=3$.

five deepest valleys within the sampling area [30]. By using the $S_{10 z}$ instead of the $S_{z}$ which, in the MeX ${ }^{\circledR}$ software, is the maximum vertical distance between the highest peaks and the lowest valleys, the influence of extreme outliers is reduced. Theoretical studies of the $2 \mathrm{D}$-version of the $\mathrm{S}_{\mathrm{a}}$ parameter, $\mathrm{R}_{\mathrm{a}}$, have demonstrated limitations of separating between surfaces with different appearances [35, 40]. To discriminate between surfaces with the same $R_{a}$ or $S_{a}$ values, the kurtosis and skewness $\left(\mathrm{R}_{\mathrm{sk}}\right.$ and $\mathrm{R}_{\mathrm{ku}}$ in $2 \mathrm{D}$ and $\mathrm{S}_{\mathrm{sk}}$ and $\mathrm{S}_{\mathrm{ku}}$ in 3D) have been suggested as complementing parameters $[35,40]$. These parameters describe the sharpness and distribution of peaks and valleys of the surface and are defined from the height distribution curve of the profile/surface (see Fig. 3). A surface with skewness above zero and kurtosis above three consists of more and/or higher peaks than valleys. Since the $S_{\mathrm{sk}}$ and $S_{\mathrm{ku}}$ parameters are calculated by using the surface height $(\eta)$ to a power of 3 and 4 , respectively (see Table $\mathbf{1}$ ), outliers in the surface topography will influence the parameters significantly. $S_{\mathrm{sk}}$ and $S_{\mathrm{ku}}$ are both moderately affected by the sampling interval [30].

\subsubsection{Spatial Parameters}

Spatial parameters describe the texture of the surface such as randomness and periodicity. The autocorrelation length, $S_{\mathrm{al}}$, shows if the surface is dominated by high (small $S_{\mathrm{al}}$ value) or low (high $\mathrm{S}_{\mathrm{al}}$ value) frequency surface features and was chosen to give information on different sub-levels of the surfaces. $S_{a l}$ is independent of the sampling interval. The other spatial parameter chosen was the texture aspect ratio $\left(\mathrm{S}_{\mathrm{tr}}\right)$. This parameter identifies topographic texture patterns present in any direction of the surface. A $\mathrm{S}_{\mathrm{tr}}>0.5$ indicates a surface with strong uniform texture in all directions while a $S_{t r}<0.3$ indicates an anisotropic surface [28]. The $S_{\text {tr }}$ parameter is somewhat affected by the sampling interval but the texture character is not altered [28].

\subsubsection{Hybrid Parameters}

Hybrid parameters describe a combination of spatial and amplitude characteristics, where a change in either of these two may induce changes in the hybrid parameters [28]. Of the spatial parameters, the root-mean-square slope of the surface $\left(\mathrm{S}_{\mathrm{dq}}\right)$ and the developed interfacial area ratio $\left(\mathrm{S}_{\mathrm{dr}}\right)$ were chosen for evaluation. $S_{\mathrm{dr}}$, is obtained by calculating the topographical area with respect to the reference plane and gives the surface enlargement induced by the different pretreatments. For rough surfaces an $S_{d r}$ value above $1 \%$ is usually obtained, while for very steep surfaces, $S_{d r}$ values above $10 \%$ are commonly obtained [28].

The $S_{\mathrm{dq}}$ parameter is the equivalent of the mean slope parameter in 2D analysis and gives information about the corrugation of the surface. This parameter can be used to estimate the interface shear strength [37].

\subsubsection{Volume Parameters}

The surface volume parameters are all defined from the Bearing Area Ratio of the surface (Fig. 4). The parameters chosen were the material $\left(\mathrm{V}_{\mathrm{mc}}\right)$ and void volume $\left(\mathrm{V}_{\mathrm{vc}}\right)$ in the core zone together with the peak material $\left(\mathrm{V}_{\mathrm{mp}}\right)$ and valley void volume $\left(\mathrm{V}_{\mathrm{vv}}\right)$ of the bearing area curve of the topography. To calculate these parameters, the bearing area

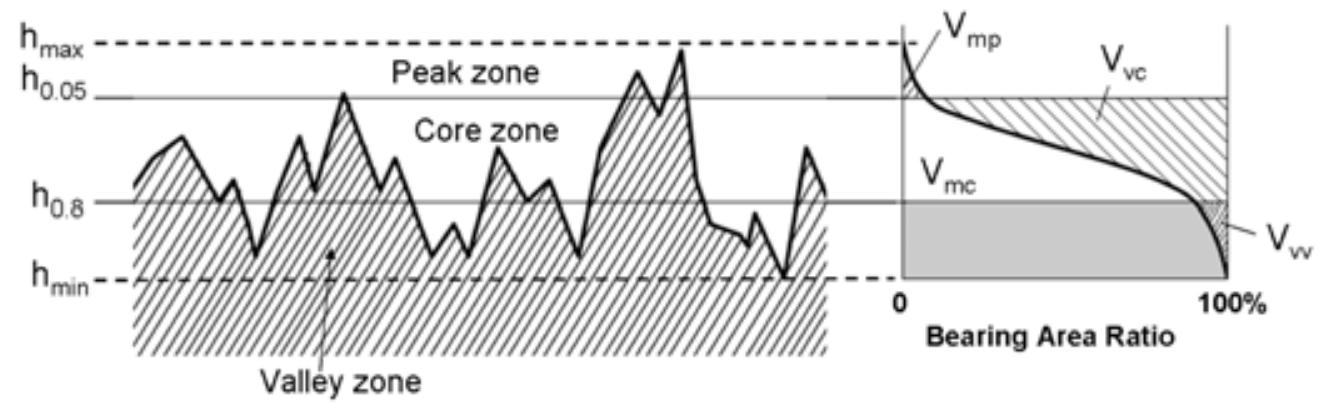

Fig. (4). Schematic description of the volume parameters defined from the bearing area ratio. 
ratio is divided into peak, core and valley zones by horizontal lines parallel to the average line at $5 \%$ and $80 \%$ in the Bearing Area Ratio curve, respectively [30]. The parameters were chosen because of their ability to visualise changes in bearing at different levels of the surface. The bearing of the surface may be correlated with the biomechanical properties of the surface and the long term anchoring of the implant.

\section{RESULTS}

All surfaces have been analysed at different magnifications/scan sizes and by applying filters of different cut-off wavelengths. Through this procedure, topographical information of surface features corresponding to different spatial levels has been collected. To visualise changes in parameter values with different magnification and filter sizes, the parameter values are plotted as a function of filter size. The unfiltered data at the largest magnification for 3DSEM $(\times 500)$ and AFM $(10 \times 10 \mu \mathrm{m})$ are also included in the plots.

The parameter values for the $1 \%$ filter size turned out to be afflicted with large errors (presumably due to limitation in the resolution) and are therefore not included in the analysis. Thus, the smallest surface features that can be detected fall in the range of 150 to $1.5 \mathrm{~nm}$. In general, the data presented are obtained from three different spots on the surface and lines are presented expressively to guide the eye. The result section is structured as follows. For each set of parameters a comparison between the physically modified (turned and blasted) surfaces is made followed by a comparison highlighting the effect of the chemical modification. The latter part is divided between blasted and non-blasted surfaces.

\subsection{Surface Average Height}

The $S_{a}$ and $S_{q}$ parameters are strongly correlated (see Table 1), which gives plots with the same appearance. Since the $S_{a}$ parameter is more widely used in the dental implant literature, it was chosen to illustrate the dependence on filter size (Fig. 5). The $S_{a}$ value increases with increasing filter size for all surfaces and the TS surface is well separated from the blasted surfaces over the entire filter size range. For filter sizes above $5 \mu \mathrm{m}$ it is also possible to discriminate between the fine blasted (FB) and coarse blasted (CB) surfaces. The parameter values in this filter size range are obtained from all applied filter sizes in the $\times 500$ 3D-SEM magnifications and from the 10,15 , and $20 \%$ filter sizes in the $\times 12003$ D-SEM magnification.

With the $\mathrm{S}_{\mathrm{a}}\left(\right.$ or $\mathrm{S}_{\mathrm{q}}$ ) parameter it is not possible to observe changes due to the chemical treatment at any filter size or magnification (compare the curves for $\mathrm{CB}$ and $\mathrm{CB}+\mathrm{HF}$ in Fig. (5)). However, differences between the two surfaces are clearly observed in the SEM images shown in Fig. (1). The fact that the calculated $S_{a}$ value is unable to reveal the differences seen in the analogue image shows the limitation in digitalising the images. By using AFM, smaller surface features become accessible and a clear distinction between the TS and TS+HF surface is seen in the calculated $S_{a}$ value (Fig. 5). The unfiltered values obtained from the $10 \times 10 \mu \mathrm{m}$ scan size in AFM fall on the same line as the values from
3D-SEM for the same surfaces. The values are encircled in Fig. (5). The HF etching clearly introduces changes in the surface topography at the sub-micrometre to nanometre level (see Fig. 1).

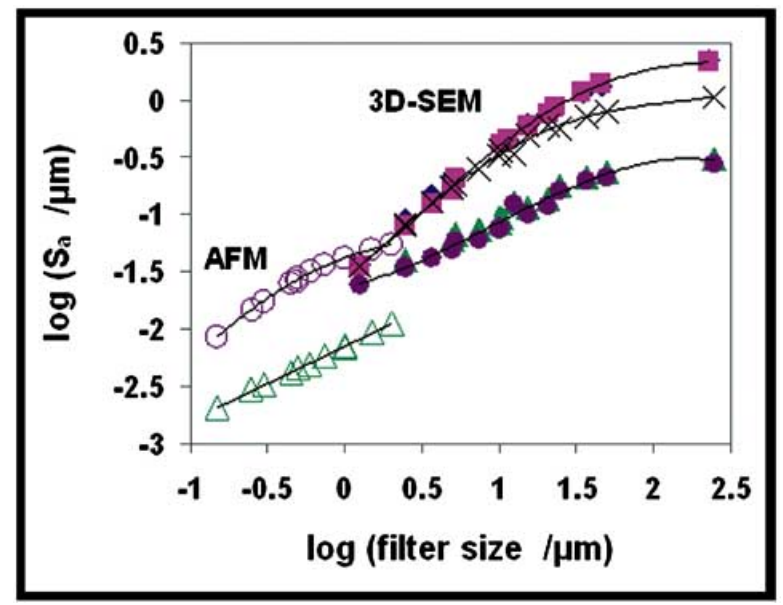

Fig. (5). $\log \left(\mathrm{S}_{\mathrm{a}}\right)$ as a function of $\log ($ filter size $/ \mu \mathrm{m})$ for all surfaces analysed. Values obtained from the 3D-SEM techniques are plotted as filled symbols while AFM results are shown as empty symbols. The lines are presented expressively to guide the eye. The unfiltered $\mathrm{AFM}$ data are encircled in the figure. $\downarrow \mathrm{CB}, \boldsymbol{\square}=\mathrm{CB}+\mathrm{HF}, \times=\mathrm{FB}$, $\boldsymbol{\Delta}=\mathrm{TS}, \bullet=\mathrm{TS}+\mathrm{HF}$. Unfilled symbols represent AFM data.

\subsection{Skewness and Kurtosis}

From the qualification tests made with the $\mathrm{MeX}^{\mathbb{B}}$ software, the skewness $\left(\mathrm{S}_{\mathrm{sk}}\right)$ parameter was shown to be affected by instrumental settings and large variations in $\mathrm{S}_{\mathrm{sk}}$ were obtained for the same surface. Fig. (6) shows the $S_{\text {sk }}$ parameter values for the $\mathrm{CB}$ and $\mathrm{FB}$ surfaces, where the rings and triangles are values from the three analysed spots and the line is the average of all obtained values. For the CB surface the $S_{\text {sk }}$ values are around zero (Gaussian distribution) but changes from positive to negative values with increasing filter size. In contrast, the $S_{\text {sk }}$ parameter is negative over the entire filtering range for the FB surface. A negative value for $\mathrm{S}_{\mathrm{sk}}$ points at a surface with more and/or deeper valleys than peaks.

The appearance of the kurtosis curve is similar to that of the skewness curve. Fig. (7) shows $S_{k u}$ values obtained from all three spots on the $\mathrm{CB}$ surface. Also in this case a large spread in parameter values is observed. The $\mathrm{S}_{\mathrm{ku}}$ value decreases from about 6 to 3.5 as the filter size is increased. For a Gaussian surface, the $S_{\mathrm{ku}}$ value is 3 and the larger values found for the CB surface show a peaky surface even though the $S_{\text {sk }}$ values were grouped around zero indicating a Gaussian distribution. Although the variation in the $S_{k u}$ parameter for the same surface was quite large for all surfaces, some trends could be seen. In Fig. (8) the $S_{k u}$ values for all blasted surfaces are plotted together. The $\mathrm{CB}$ and $\mathrm{CB}+\mathrm{HF}$ surfaces are overlapping with decrease in the $\mathrm{S}_{\mathrm{ku}}$ with increased filter size. For the FB surface the $S_{k u}$ parameter is independent of filter size with a value around 3.8. Thus, also for the FB surface some peakedness is observed. This is in agreement with the negative value of the skewness parameter since more and/or deeper valleys will lead to sharper peaks. 


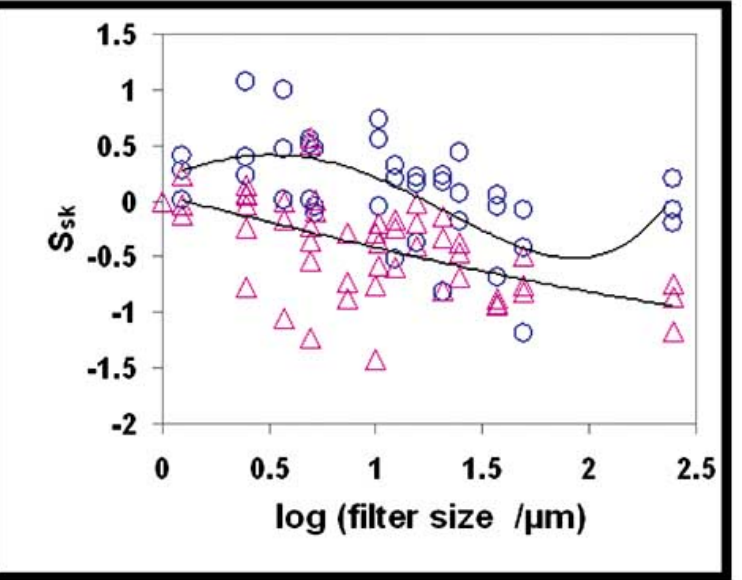

Fig. (6). Skewness $\left(S_{\text {sk }}\right)$ values obtained for the coarse $(O=C B)$ and fine $(\Delta=\mathrm{FB})$ blasted surfaces by the 3D-SEM technique. Symbols represent single values obtained on all three analysed areas and the line represents the average.

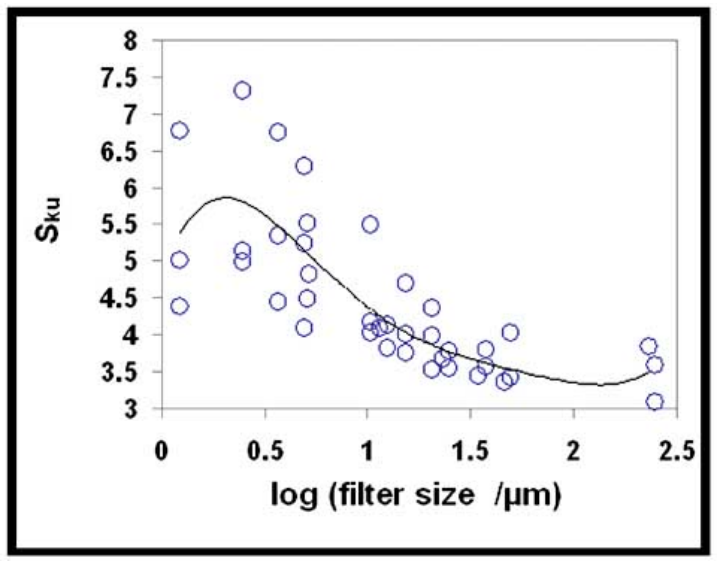

Fig. (7). Kurtosis $\left(S_{\mathrm{ku}}\right)$ values for the coarse blasted surface $(\mathrm{CB})$. Rings represent single values obtained on all three analysed areas and the line represents the average.

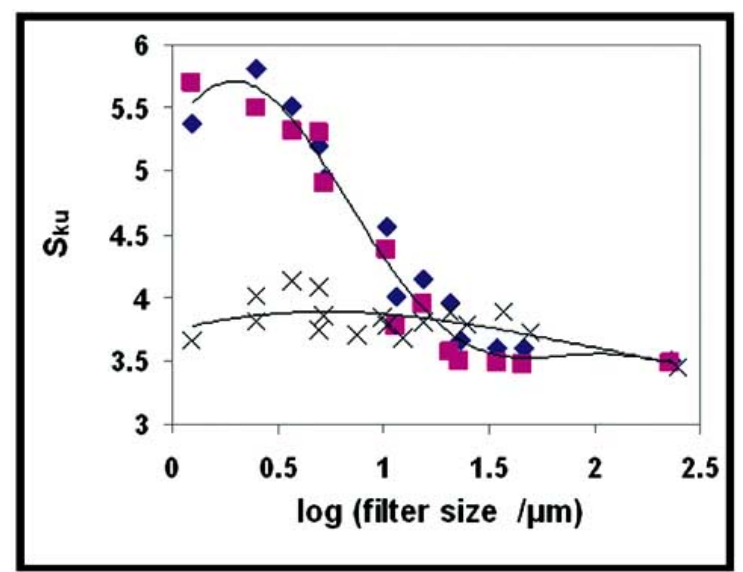

Fig. (8). Average kurtosis $\left(S_{\mathrm{ku}}\right)$ values (3D-SEM technique) for the blasted surfaces. The fine and coarse blasted surfaces are separated at lower filter sizes. No separation was obtained between the blasted and chemically treated surfaces. $\downarrow \mathrm{CB}, \boldsymbol{\square}=\mathrm{CB}+\mathrm{HF}, \times=$ FB.
No change was observed after the chemical treatment of the turned and blasted surfaces probably due to the large spread in the $S_{\mathrm{sk}}$ and $S_{\mathrm{ku}}$ values. This also applies to the measurements with AFM.

\subsection{Maximum Surface Height}

Fig. (9) shows the $S_{10 z}$ average values for the CB, FB and TS surfaces. As expected, the lowest $S_{10 z}$ values were obtained for the TS surface followed by the FB and $\mathrm{CB}$ surfaces. With the $\mathrm{S}_{10 \mathrm{z}}$ parameter it is possible to discriminate between the fine and coarse blasted surfaces over the whole 3D-SEM range with slightly increased separation with increasing filter size. However, no change in the $S_{10 z}$ parameter was observed after the HF etching of the $\mathrm{CB}$ and TS surfaces using 3D-SEM, Fig. (10). With the AFM technique a better resolution between the TS and TS+HF surfaces could be obtained. The values obtained by AFM and 3D-SEM are similar for the TS+HF surface but for the untreated TS surface the values obtained by the AFM technique are significantly lower. This is probably due to differences between the two techniques and will be further discussed in section 5.1.

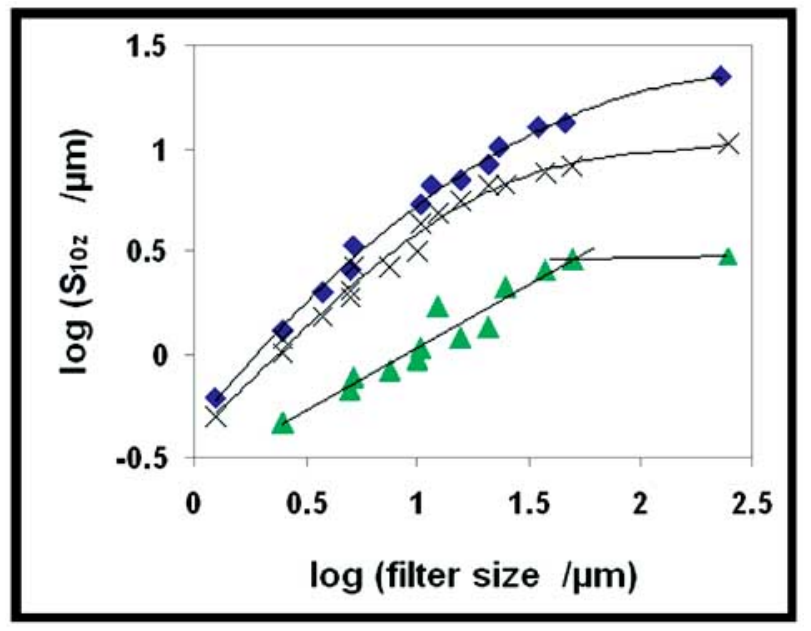

Fig. (9). Average $\log \left(\mathrm{S}_{10 \mathrm{z}}\right)$ values for the fine and coarse blasted (FB and CB) surfaces together with the turned surface (TS). Separation is achieved between all three surfaces over the whole SEM range. $=\mathrm{CB}, \times=\mathrm{FB}, \boldsymbol{\Delta}=\mathrm{TS}$.

\subsection{Autocorrelation Length}

Fig. (11) shows the autocorrelation length parameter, $S_{a l}$, for the TS and TS+HF surfaces over the entire filter size range including both AFM and 3D-SEM measurements. A linear relationship is observed for all surfaces with a slope close to one, i.e. $S_{a l}$ is directly proportional to the filter size. This illustrates that the frequency of the measured roughness is defined by specifying the filter size, i.e the surface roughness is self-affined. Thus, it is important to measure surface roughness parameters as a function of filter size to fully describe the surface.

\subsection{Texture Aspect Ratio}

The $S_{\text {tr }}$ parameter gives information about directional texture or randomness of the surface $[28,30]$. A value below 0.3 indicates the presence of a directional texture while a 


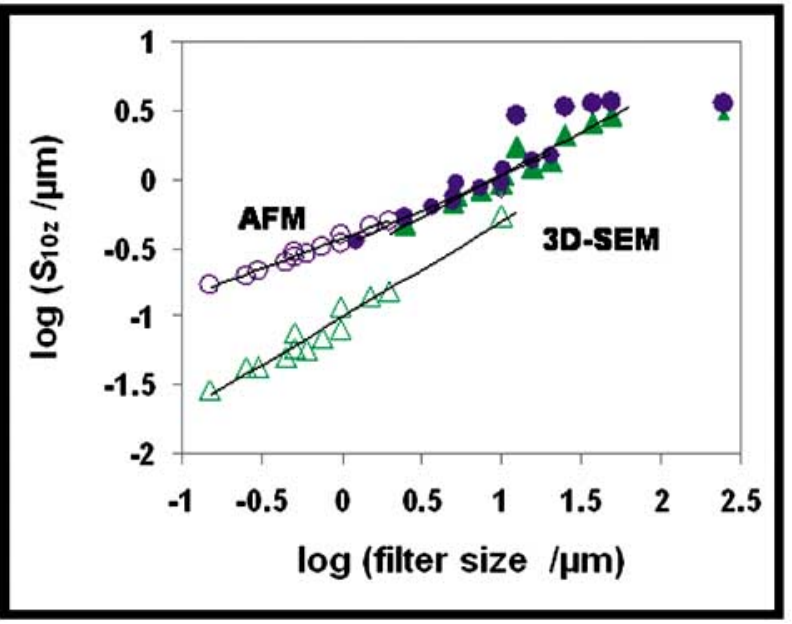

Fig. (10). Average $\log \left(\mathrm{S}_{10 \mathrm{z}}\right)$ values for the turned surfaces with and without HF treatment. No separation is achieved within the SEM range whereas higher values are obtained for the etched surface within the AFM range. $\boldsymbol{\Delta}=\mathrm{TS}, \bullet=\mathrm{TS}+\mathrm{HF}$. Unfilled symbols represent AFM data.

value above 0.5 indicates a random surface structure. Fig. (12) shows the $S_{\text {tr }}$ values for the turned and blasted surfaces. The coarse blasted (CB) surface shows a transition to directional texture at the largest magnifications. The opposite is seen for the turned surface (TS) which shows directional texture for the larger filter sizes (low magnification) but approaches a more random surface structure at the smaller filter sizes. A small effect of chemical treatment was observed at the largest magnifications; see the encircled area in Fig. (12). In order to explain these results, the plots are evaluated together with SEM and AFM images.

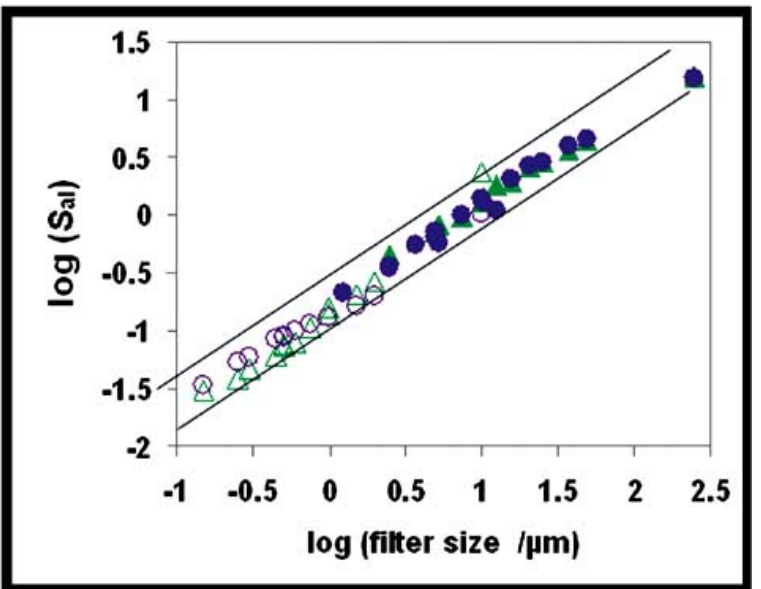

Fig. (11). Average $\log \left(\mathrm{S}_{\mathrm{al}}\right)$ values for all surfaces and both techniques. The slope of the line is close to one. $\boldsymbol{\Delta}=\mathrm{TS}, \bullet=$ TS + HF. Unfilled symbols represent AFM data.

In Fig. (13) the SEM images for the FB and CB surfaces are compared. Three different magnifications are used together with three filter sizes in order to cover the whole $3 \mathrm{D}-\mathrm{SEM}$ filter size range. For both surfaces the $\mathrm{S}_{\text {tr }}$ values are above 0.5 in the lower magnification region and were identified as random structure (Fig. 12). However, at the highest magnifications, texture was found for the $\mathrm{CB}$ surface but not for the FB surface. Blasting with larger particle size creates surface features of larger size, which can be seen at the higher magnifications in the SEM images in Fig. (13). The larger features may still have unaffected parts with a reminiscence from the turned surface. Another possible explanation is that at high magnifications only few surface features are analysed on the $\mathrm{CB}$ surface which can give an apparent directional texture. For the FB surface the features created are smaller and turning tracks are thus completely destroyed leading to a random surface also at the highest magnifications. Furthermore, the greater number of surface features reduces the probability of obtaining a false directional pattern. For the coarse blasted and chemically etched surface $(\mathrm{CB}+\mathrm{HF})$ the small features created somewhat masks the turning tracks and the surface is less textured. As expected, the TS surface is textured at large filter sizes due to the turning tracks on the surface. This is clearly seen in the SEM images in Fig. (14). When filter sizes are decreased, the turning tracks disappear which explains the increase in $\mathrm{S}_{\mathrm{tr}}$ value at the highest magnification, see the $5 \%$ filter size for the two largest magnifications.

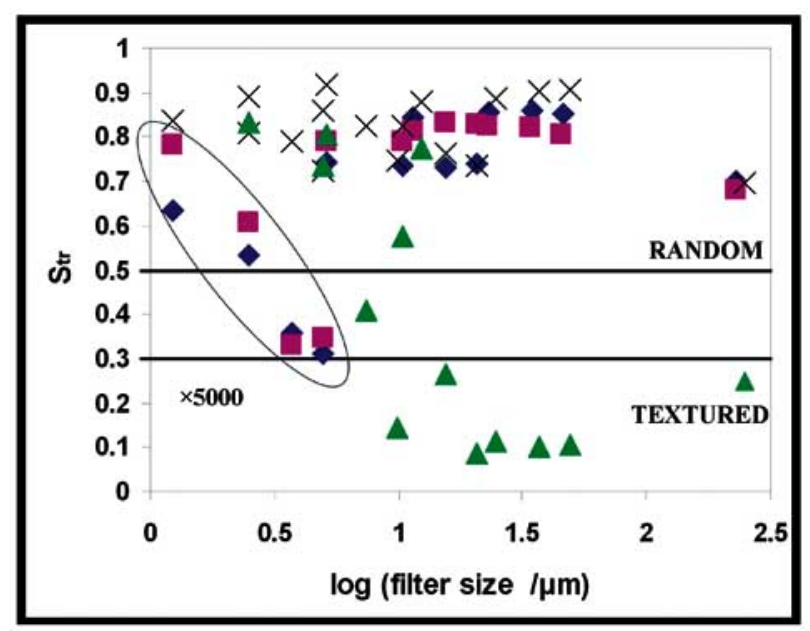

Fig. (12). Average texture aspect ratio $\left(\mathrm{S}_{\mathrm{tr}}\right)$ for the $\mathrm{FB}, \mathrm{CB}, \mathrm{CB}+\mathrm{HF}$ and TS surfaces. Randomness is obtained for the FB surface over the whole SEM range while texture can be seen for the $\mathrm{CB}$ and $\mathrm{CB}+\mathrm{HF}$ surfaces at the highest magnification (lowest filter sizes). The turned surface is classified as textured surface at the higher filter sizes and goes towards a random structure at the lower filter sizes. $=\mathrm{CB}, \boldsymbol{\|}=\mathrm{CB}+\mathrm{HF}, \times=\mathrm{FB}, \boldsymbol{\Delta}=\mathrm{TS}$.

Fig. (15) shows the $S_{\text {tr }}$ parameter for the TS and TS $+H F$ surfaces for both 3D-SEM and AFM. In the AFM analysis the $S_{\text {tr }}$ values for the two surfaces are well separated, clearly showing the effect of the HF etching. In the 3D-SEM analysis similar $\mathrm{S}_{\mathrm{tr}}$ values are obtained for the two surfaces with a clear texture at large filter sizes and an apparent random surface when the filter size is decreased. Since AFM has a higher resolution the difference in the values obtained with the two techniques shows the limitation of the 3D-SEM technique at the larger magnifications. In AFM the TS surface is textured in the entire filter size range while the $\mathrm{TS}+\mathrm{HF}$ surface is random. This is also clearly seen in the AFM images shown in Fig. (16). For the TS surface the turning tracks are observed also at the highest magnification and smaller filter size $(3 \times 3 \mu \mathrm{m}, 5 \%)$. The small features created in the chemical pre-treatment are clearly seen in the AFM images and these precipitates effectively mask the turning tracks and the surface appears random. 


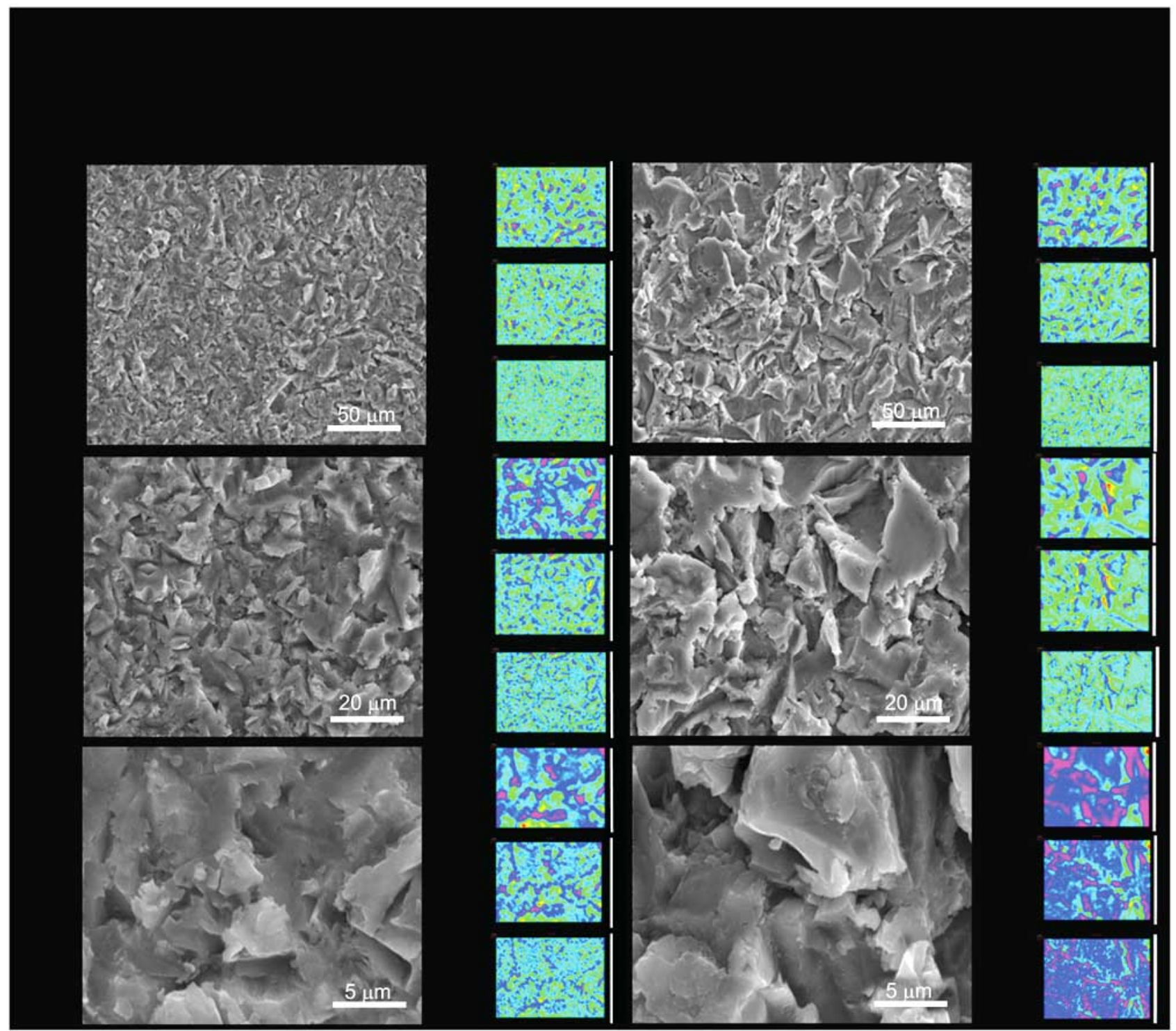

Fig. (13). The FB and CB surfaces at three different SEM magnifications together with filtered MeX ${ }^{\circledR}$ images after application of filter with sizes $20 \%, 10 \%$, and $5 \%$ of the horizontal image width. The depth scale of the $\mathrm{MeX}^{\circledR}$ filter images goes from purple which represents the deepest, through blue, green, and yellow up to red which represents the highest points on the surface. The depths are all different in the images. As seen in the filtered images, the fine blasted surface (FB) consists of smaller surface features than the coarse blasted surface (CB).

\subsection{Hybrid Parameters}

The hybrid parameters are significantly affected by the sampling interval [30] which is changed with magnification and scan size of the SEM and AFM technique. This results in changes in absolute parameter values but the trend for the different surfaces is the same. This is showed by the $S_{\mathrm{dr}}$ parameter at $\times 500$ magnification $3 \mathrm{D}$ in Fig. (17). For the blasted surfaces $S_{\mathrm{dr}}$ increases with increasing filter size while smaller changes are observed for the turned surface. There is a clear difference between the TS, FB and CB surfaces with high values for the blasted surfaces and a much lower value for the turned surface. The HF etching increases the $S_{d r}$ value slightly for the blasted sample but has a profound influence on the value for the turned surface (Fig. 17). Note that after the chemical treatment of the turned surface the $S_{d r}$ values are similar to those of the fine blasted surface. Preliminary results show that the $S_{\mathrm{dq}}$ parameter can be used to estimate the interface shear strength [37] and it is therefore important to include this parameter in characterising surface roughness.

The results obtained for the $S_{\mathrm{dr}}$ and $S_{\mathrm{dq}}$ parameters actually indicate that changes on very small levels can be recorded in the 3D-SEM method. This is an important finding and will be further discussed in section 5.2.

\subsection{Volume Parameters}

All volume parameters show the same dependence on filter size for all surfaces and the relationship between the surfaces is similar to the relationship seen for the $S_{a}$ and $S_{q}$ parameters. For visualisation, the $\mathrm{V}_{\mathrm{vc}}$ (void volume in core material) was chosen and is presented in Fig. (18). The 


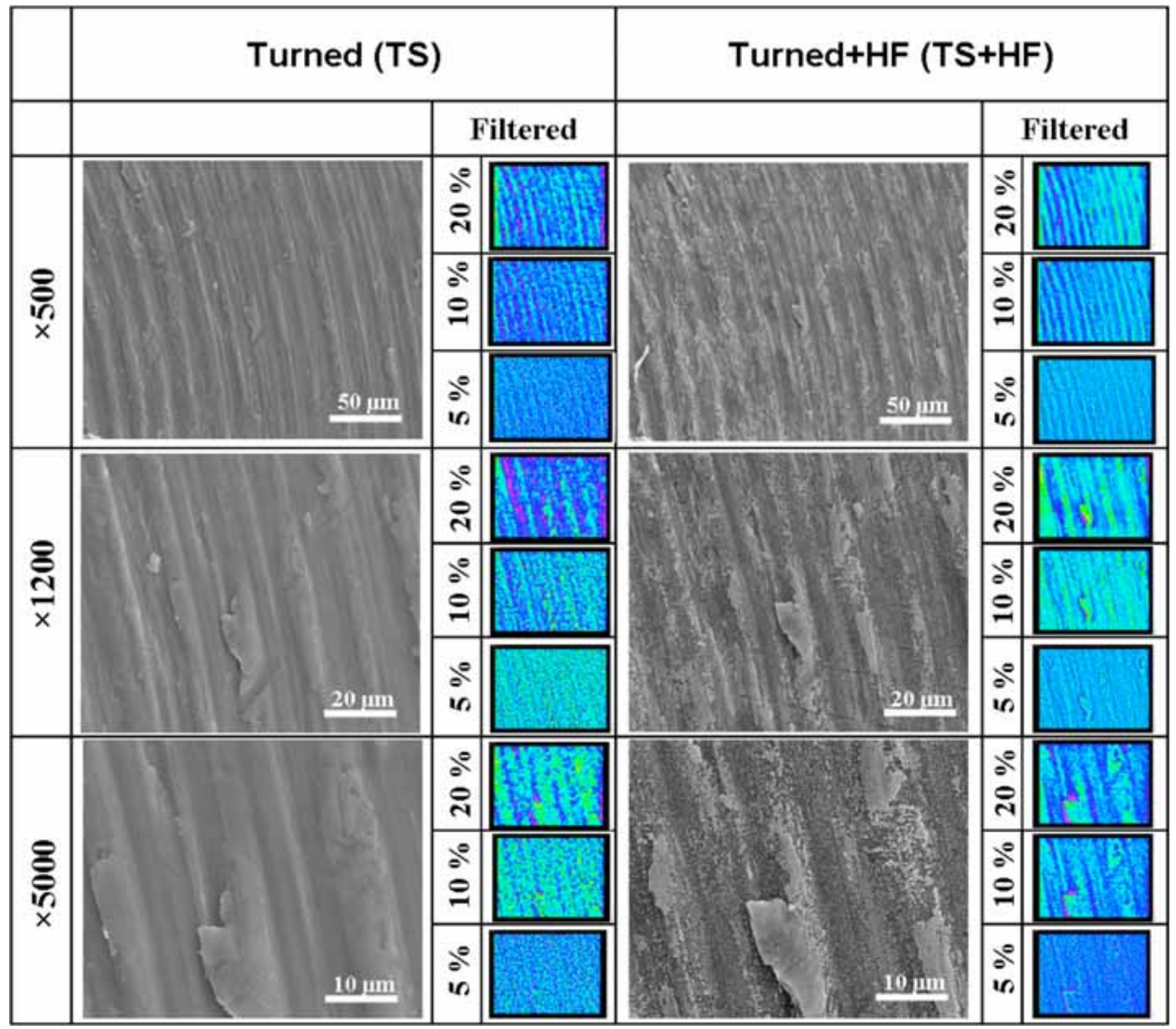

Fig. (14). SEM images collected at three different magnifications for the turned surfaces with and without HF treatment (TS and TS+HF) together with filtered $\mathrm{MeX}^{\circledR}$ images after application of filter with sizes $20 \%, 10 \%$, and $5 \%$ of the horizontal image width. Differences between the two surfaces become more visible with increased magnification. The $\mathrm{MeX}^{\circledR}$ filtered images show quite clearly that the turning tracks are erased more and more by the application of smaller filter sizes.

values for the $\mathrm{V}_{\mathrm{vc}}$ are well separated for the TS, FB and CB surfaces. For the latter two, similar values are obtained for filter sizes less than $5 \mu \mathrm{m}$ which is similar to the findings for the $S_{a}$ parameter. The $V_{v c}$ is not affected by HF etching on coarse blasted and turned surfaces in the 3D-SEM filter size range. However, in AFM a clear difference in the $V_{v c}$ values is observed after HF etching of the turned surface (Fig. 18). Unfiltered data at $10 \times 10 \mu \mathrm{m}$ scan size for both surfaces are in the same range as the data obtained with comparable filter size in the 3D-SEM technique (data encircled in Fig. (18)).

\section{DISCUSSION}

In this study 3D surface roughness parameters were critically evaluated when used as a measure for describing dental implant surfaces. A variable scale analysis was employed by measuring the surface roughness parameters as a function of magnification and filter size. The results show that the two techniques used complement each other and the discussion will start with a comparison between the results obtained by the two techniques. In addition to the more commonly used surface height parameters, it was found that the parameters describing spatial, hybrid and volume properties were useful for describing the surface topography and they are further discussed below together with the use of functional parameters. The variable scale analysis used is further discussed in relation to the fractal behaviour observed and the usefulness of calculating the fractal dimension and crossover size. The discussion section ends with a summary of literature in vivo data and the correlation with surface roughness parameters. 


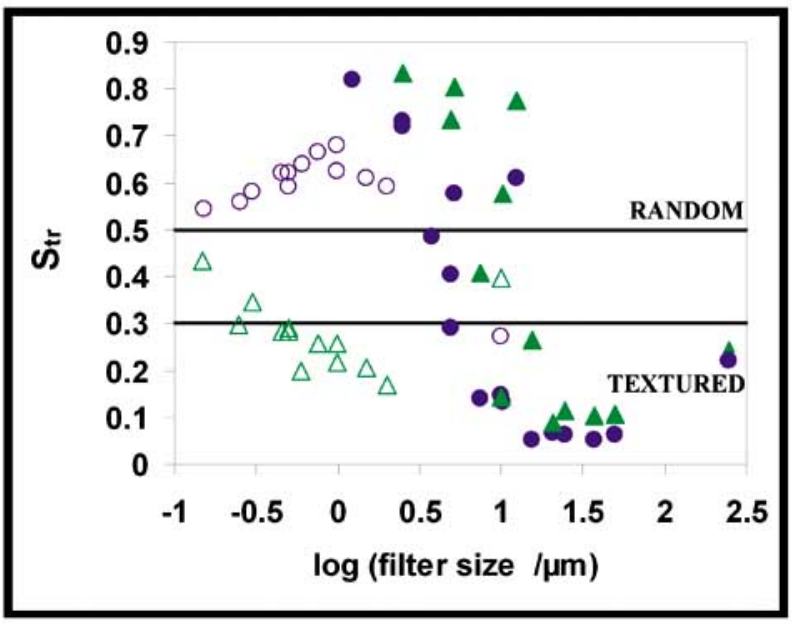

Fig. (15). Average $S_{\text {tr }}$ parameter values for the turned surfaces with and without HF treatment for both characterisation techniques. Both surfaces go from random to textured surfaces with increased filter size within the SEM range. For the AFM range, the chemically etched surface is classified as a random surface over the whole range while the untreated surface goes from the mixed zone to textured surface with increased filter size. $\boldsymbol{\Delta}=\mathrm{TS}, \bullet=\mathrm{TS}+\mathrm{HF}$. Unfilled symbols represent AFM data.

\subsection{Comparison Between 3D-SEM and AFM}

In Table $\mathbf{2}$ an overview of the surface roughness parameters ability to discriminate between the different surfaces is given. By the 3D-SEM technique, the fine-, and coarse blasted surfaces were separated at the larger filter sizes in ten of the thirteen parameters evaluated (denoted as Partly in Table 2). With these ten parameters, the turned surface was well separated from the blasted surfaces in the entire filter size range. By the AFM technique the TS and $\mathrm{TS}+\mathrm{HF}$ surfaces were well separated by ten of the evaluated parameters, while with the 3D-SEM technique it was not possible to separate the chemically treated and untreated surfaces (Table 2).

For most of the parameters, differences between values calculated from the AFM and 3D-SEM techniques are observed (Figs. 5, 10, 15). The different values obtained may originate from a difference in the size of the area analysed, reflected in the filter size, or differences in the measurement techniques [34]. In 3D-SEM, a grey-scaled image is obtained by electron bombardment and converted to a matrix of data points used for calculating the surface roughness parameters. The AFM technique is a scanning probe technique where a probe is mechanically scanned over the surface and topographical height information is obtained from the force

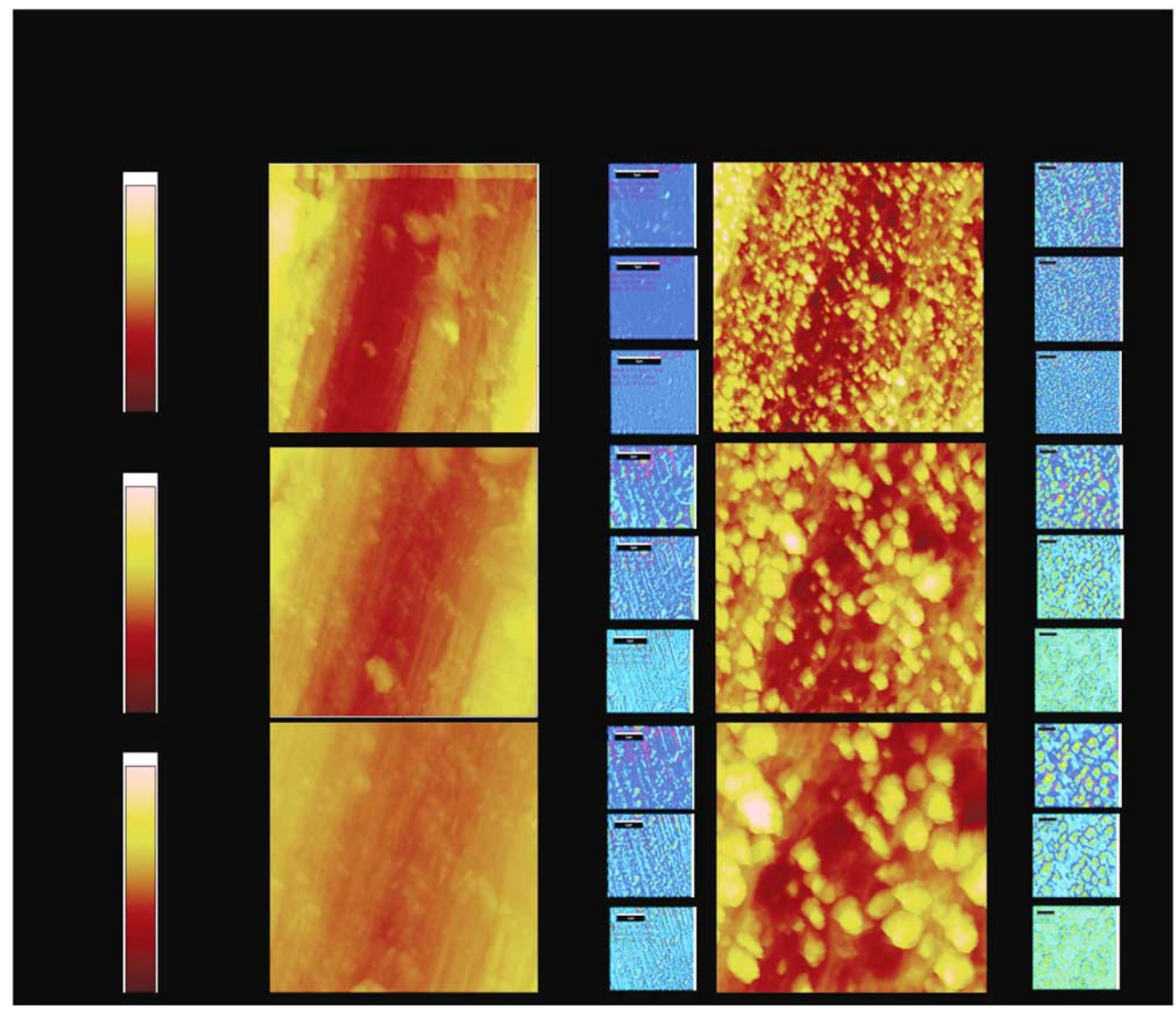

Fig. (16). AFM images collected at the three different scan sizes of the turned surface with and without HF treatment (TS and TS+HF), together with filtered $\mathrm{MeX}^{\circledR}$ images after application of filter of sizes $20 \%, 10 \%$, and $5 \%$ of the horizontal image width. The chemical treatment induces a topography consisting of small peaks which covers the surface. By application of filters, the turning tracks are erased and a very smooth surface is created for the turned surface (TS). 
between the tip and the surface. In AFM the sensitivity is dependent on the tip size in relation to the roughness and in 3D- SEM the limiting factors are the quality of the image and the resolution.

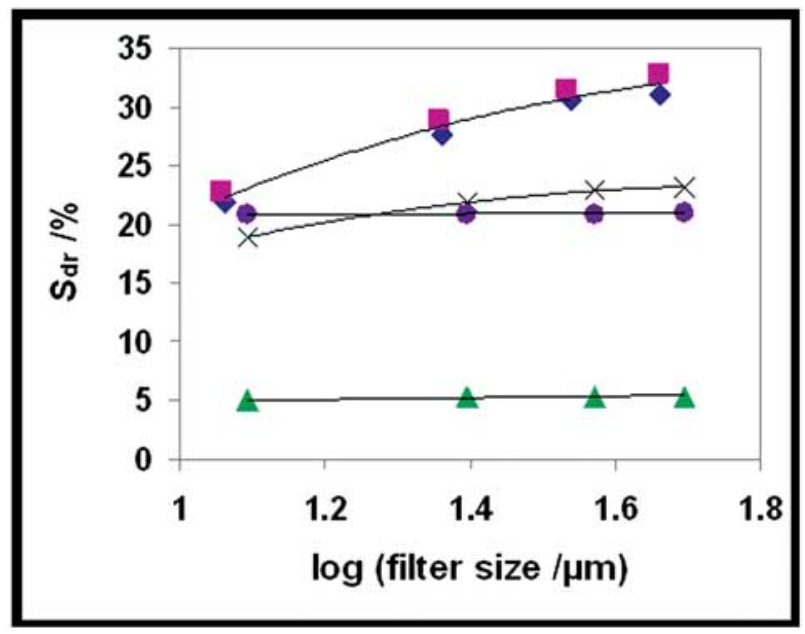

Fig. (17). Average $\log \left(\mathrm{S}_{\mathrm{dr}}\right)$ values for all surfaces at the lowest SEM magnification $(\times 500)$. A similar relationship was obtained for the $\mathrm{S}_{\mathrm{dq}}$ parameter. $\bullet=\mathrm{CB}, \boldsymbol{\mathbf { a }}=\mathrm{CB}+\mathrm{HF}, \times=\mathrm{FB}, \boldsymbol{\Delta}=\mathrm{TS}, \bullet=$ TS+HF. Unfilled symbols represent AFM data.

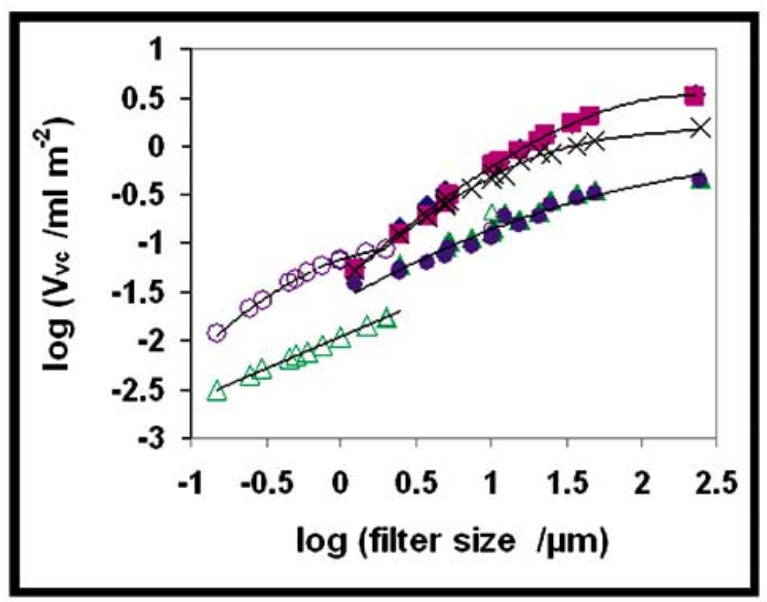

Fig. (18). Average $\log \left(\mathrm{V}_{\mathrm{vc}}\right)$ values for all surfaces obtained using AFM and 3D-SEM. Similar relationships were obtained for all volume parameters. $\bullet=\mathrm{CB}, \boldsymbol{\square}=\mathrm{CB}+\mathrm{HF}, \times=\mathrm{FB}, \boldsymbol{\Delta}=\mathrm{TS}, \bullet=$ TS+HF. Unfilled symbols represent AFM data.

In this study, the SEM images and AFM data were imported into the $\mathrm{MeX}^{\circledR}$ software where parameters were calculated. The pixel distance used in the SEM image or AFM scan gives the lowest resolution limit possible of the images in the $\mathrm{MeX}^{\circledR}$ software. Although the pixel distance for SEM $\times 2500$ and $\times 5000$ images and AFM $10 \times 10 \mu \mathrm{m}$ and $5 \times 5 \mu \mathrm{m}$ scan sizes are similar, it was not possible to distinguish between the TS and TS+HF surfaces using 3DSEM together with the $\mathrm{MeX}^{\circledR}$ software. This shows that the small surface features induced by the chemical etching are below the detection limit for 3D-SEM. The resolution limit for 3D-SEM together with the $\mathrm{MeX}^{\circledR}$ software could be affected by various parameters such as: brightness and contrast of the images, disturbances from outside vibrations not allowing for sufficient magnification or resulting in blurry images, imperfectly overlapping stereo-SEM pair resulting in loss of sharpness of the 3D-models and shadowing effects masking some parts of the area. In the qualification of the method, the effect of brightness and contrast on the calculated roughness parameters was evaluated and only if very bright or dark images were collected an effect on the parameters was obtained. Blurry images will give a contribution to the parameter values related to the height resolution for different magnifications and filter sizes.

Table 2. Overview of the Surface Roughness Parameters Ability to Discriminate Between the Analysed Surfaces

\begin{tabular}{|c|c|c|c|c|}
\hline Surfaces & TS, FB, CB & CB, CB+HF & \multicolumn{2}{|c|}{ TS, TS+HF } \\
\hline \hline Technique & $3 D-S E M$ & $3 D-S E M$ & $3 D-S E M$ & $A F M$ \\
\hline $\mathbf{S}_{\mathbf{a}}$ & Partly & No & No & Yes \\
\hline $\mathbf{S}_{\mathbf{q}}$ & Partly & No & No & Yes \\
\hline $\mathbf{S}_{\mathbf{s k}}$ & No & No & No & No \\
\hline $\mathbf{S}_{\mathbf{k u}}$ & No & No & No & No \\
\hline $\mathbf{S}_{\mathbf{1 0 z}}$ & Partly & No & No & Yes \\
\hline $\mathbf{S}_{\mathbf{a l}}$ & No & No & No & No \\
\hline $\mathbf{S}_{\mathbf{t r}}$ & Partly & No & No & Yes \\
\hline $\mathbf{S}_{\mathbf{d r}}$ & Partly & Partly & Yes & Yes \\
\hline $\mathbf{S}_{\mathbf{d q}}$ & Partly & Partly & Yes & Yes \\
\hline $\mathbf{V}_{\mathbf{m p}}$ & Partly & No & No & Yes \\
\hline $\mathbf{V}_{\mathbf{m c}}$ & Partly & No & No & Yes \\
\hline $\mathbf{V}_{\mathbf{v c}}$ & Partly & No & No & Yes \\
\hline $\mathbf{V}_{\mathbf{v v}}$ & Partly & No & No & Yes \\
\hline
\end{tabular}

Yes: Separation was achieved over the entire filter range.

No: No separation was achieved.

Partly: Separation was achieved at some filter sizes.

\subsection{Hybrid Parameters}

The hybrid parameters, $\mathrm{S}_{\mathrm{dr}}$ and $\mathrm{S}_{\mathrm{dq}}$, were the only parameters able to distinguish between the turned and etched (TS and TS + HF) surfaces within the 3D-SEM range (Table 2 and Fig. 17). These parameters are combinations of amplitude and spatial characteristics of the surface [28]. With $\mathrm{S}_{\mathrm{dq}}$ for example, the derivative with respect to the surface height is obtained, which is more sensitive to changes at all levels than the amplitude parameters $S_{q}$ or $S_{a}$. Since none of the amplitude or spatial parameters alone were able to distinguish between the TS and TS+HF surface, this shows that parameters from all groups (amplitude, spatial and hybrid) need to be used to be able to distinguish between different surfaces.

Low values for both evaluated hybrid parameters were obtained for the turned surface (TS) compared with the etched surface $(\mathrm{TS}+\mathrm{HF})$ showing the peakedness introduced by the etching. It is interesting to note that the TS+HF surface has similar $S_{d r}$ and $S_{d q}$ values as the fine blasted (FB) surface by the 3D-SEM technique (Fig. 17). The FB surface consists of higher surface features than the TS+HF surface, increasing the $S_{d r}$ and $S_{d q}$ value according to the equations shown in Table 1. However, the $S_{d r}$ and $S_{d q}$ parameters are 
also affected by the number of surface features present and captured by each pixel of the analysed surface. Since the $\mathrm{TS}+\mathrm{HF}$ surface consists of a larger number of surface features, this will contribute to the $S_{d r}$ and $S_{d q}$ values. This explains the similar values for both the $\mathrm{TS}+\mathrm{HF}$ and $\mathrm{FB}$ surfaces.

When comparing the two analysing techniques, higher $\mathrm{S}_{\mathrm{dr}}$ values for the TS+HF surface were obtained by AFM compared with 3D-SEM, 3\% and 55\% (AFM 10×10 $\mu \mathrm{m}$ ), $5.5 \%$ and $20.6 \%(3 \mathrm{D}-\mathrm{SEM} \times 500)$, for the TS and TS+HF surfaces, respectively. Also higher $\mathrm{S}_{\mathrm{dq}}$ values were recorded with AFM. Both $S_{d r}$ and $S_{d q}$ parameters are sensitive to the pixel distance (i.e. sampling interval [30]) since a larger pixel distance works as a smoothing of the surface. In general AFM is therefore more sensitive than the 3D-SEM technique with smaller analysing area and pixel distances. However, in the present study comparable pixel distances were obtained in a few cases but the differences remained; see section 5.1 for further discussion.

\subsection{Functional Parameters}

For engineering applications functional parameters are commonly defined to describe certain properties related to wear, bearing etc. [29, 30]. The functional parameters are given in terms of the average height parameter, $S_{q}$, to eliminate the effect of roughness and highlight other surface properties. For characterising dental implants the fluid retention index, $\mathrm{S}_{\mathrm{ci}}$, has been used $[3,31] . \mathrm{S}_{\mathrm{ci}}$ can be calculated by Equation 1[29] and describes the ability of the surface to keep the fluid. This is important for tribology and might also be important for the tissue-implant contact.

$S_{c i}=\frac{V_{v c}}{S_{q}}$

The $\mathrm{S}_{\mathrm{ci}}$ parameter for the surfaces analysed in the present study was calculated and found to be between 1.1 and 1.3. This value is independent of the technique used and of magnification and filter size. For a Gaussian surface, $S_{c i}$ is 1.56 and for sand blasted and turned surfaces the $S_{c i}$ values are commonly around this value or higher. The low value obtained in the present study is probably an effect of the placement of the reference plane. As previously described, the reference plane was placed on the most even parts of the surface and for plateau type surfaces the $\mathrm{S}_{\mathrm{ci}}$ value is much lower [29].

In two recent studies, the retention strength in vivo was correlated to the $S_{\mathrm{ci}}$ index $[3,31]$. Inconsistent results were reported with increased retention strength for surfaces with high [3] and low [31] $\mathrm{S}_{\mathrm{ci}}$ index, respectively. Arvidsson et al. [31] compared different blasted surfaces as well as the surfaces of a number of commercially available implants. The $\mathrm{S}_{\mathrm{ci}}$ index for all these surfaces, except TiUnite, is very similar ranging from 1.36 to 1.57 including the turned surface as a reference. For TiUnite the $\mathrm{S}_{\mathrm{ci}}$ value falls outside this range with $\mathrm{S}_{\mathrm{ci}}=1.86$. Since different surfaces give the same $S_{\text {ci }}$ value it is questionable to correlate the $S_{\text {ci }}$ index to the in vivo performance. A better choice would be the volume parameter alone since for dental implants the entire surface is important for the in vivo performance. The volume property of a surface is known to be important when two surfaces are in close contact, for example during wear and bearing situations $[29,30]$. In the in vivo situation, the bone and implant are in close contact and the surface topography is known to affect the retention forces measured in vivo [916]. A correlation with volume parameters may therefore exist and will be discussed in Section 5.5.

\subsection{Variable Length Scale Analysis}

Surface roughness parameters are in general scaledependent and it is therefore important to perform a variable length scale analysis. For implant surfaces this scale dependence is of prime interest since roughness at different levels may contribute to the performance in vivo. For example, the physical surface treatment by blasting introduces roughness on the micrometer level and contributes to a better anchoring of the implant while chemical modifications give roughness on a smaller length scale. The fact that surfaces with the same blasting treatment but different chemical treatment show differences in performance clearly demonstrates that the finer topography introduced by the chemical treatment is important. This effect acts in parallel with any direct chemical influence introduced by changes in the surface composition or changes in the titanium dioxide properties [41]. Different methods have been proposed to accommodate the length scale dependence of surface roughness parameters including wavelet filtering, fractal analysis [34, 42-48] and Fast Fourier Transform $[22,23]$. Variable length scale analysis is applicable both to fractal and non-fractal surfaces and the fractal dimension of a surface is commonly evaluated if applicable. The analysis is based on a log-log plot of a characteristic roughness parameter versus a length scale defined as the filter size in the present work. Many natural and man-made surfaces have fractal character at least over part of the length scale and fractal analysis, being a scale independent method, has been extensively used to characterise engineering surfaces [42, 45, 48, 49]. A fractal surface can be described by a power law, exemplified here by the surface roughness parameter $S_{a}$ and the filter size, Equation (2):

$$
S_{a}(\text { filter size })=\alpha(\text { filter size })^{\beta}
$$

In the linear part of the $\log \left(\mathrm{S}_{\mathrm{a}}\right)-\log ($ filter size) plot the surface can be represented by a self-affined fractal, characterised by the fractal dimension D. From the slope of the log-log plot the fractal dimension can be calculated according to Equation (3) [42]:

$\beta=2(2-D)$

The fractal dimension is independent of filtering and has a non-integer dimension ranging from 2 for a completely flat surface to 3 representing a volume. Machined surfaces produced by more than one finishing process may have different fractal properties. The transition between two linear regions is defined by the crossover size, below which the surface is most simply described by fractal geometry. The crossover size together with the slope yields a more accurate description of the surface than either separately and has been used in the present analysis.

In the present work the changes in scale were accomplished by measuring at different magnifications and different filtering sizes. The filter size is defined in relation 
to the horizontal axis giving some overlap between different magnifications. For the amplitude and volume parameters in Figs. (5, 9, 10, 18), linear log-log relationships exist in certain filter size ranges and the fractal dimension was calculated using Equation (3). The crossover size was obtained from the crossover between the fractal behaviour (linear log-log plot) and the saturation roughness at the smallest magnification. For the TS surface, no crossover could be defined showing that for both 3D-SEM and AFM the TS surface can be represented by a self-affined fractal in the whole filter size range. In Table $\mathbf{3}$, the fractal dimensions for the different surfaces are given together with the crossover sizes. These data are based on the $S_{a}$ values but similar values are obtained when analysing the other parameters.

Table 3. Fractal Dimension and Crossover Size Determined for the Studied Surfaces

\begin{tabular}{|c|c|c|}
\hline Surface & Crossover Size $/ \boldsymbol{\mu m}$ & $\mathbf{D}$ \\
\hline \hline CB, CB+HF & 30 & 2.6 \\
\hline FB & 20 & 2.6 \\
\hline TS, TS+HF & & 2.3 \\
\hline TS_AFM & & 2.3 \\
\hline TS+HF_AFM & 1.1 & 2.6 \\
\hline
\end{tabular}

For the blasted surfaces the fractal dimension was 2.6 with a crossover of $30 \mu \mathrm{m}$ for the coarse blasted surfaces and $20 \mu \mathrm{m}$ for the fine blasted surface. The fractal dimension for the turned surface was 2.3. The chemical etching with HF did not influence the fractal dimension for the blasted and turned surface examined by 3D-SEM. However, a clear difference is observed in AFM for the TS+HF surface. The fractal dimension is the same as for the blasted surfaces but the crossover size is much smaller, $1.1 \mu \mathrm{m}$, showing the level of roughness. The fact that the fractal dimension for the TS $+\mathrm{HF}$ surface is similar to the values obtained for the FB surface is in accordance with the $S_{d r}\left(S_{d q}\right)$ values (Fig. 17). Comparing the crossover size with the AFM images in Fig. (16) reveals that the surface features are smaller than the crossover size. The fine structure in the low micron or nanometer range is often hidden by coarser contributions to the roughness and for the 3D-SEM technique the smallest features were not at all detectable, i.e. no change in the fractal dimension for the TS+HF surface and no crossover.

In order to understand the differences observed for the two techniques used, model surfaces were analysed. Fig. (19a) shows the three surfaces used with different density of features on the surface. The data were analysed in $\mathrm{MeX}^{\circledR}$ in the same way as the experimental surfaces and by an alternative way originating from fractal analysis, denoted "power law analyses" below. In the latter case the area of analysis was continuously decreased from the full size down to a few pixels and the $S_{\mathrm{a}}$-values were plotted as a function of the number of pixels $(\mathrm{N})$ on the horizontal axis in a loglog plot (Fig. 19b). The change in area was made in such a way that the same ratio between the horizontal and vertical axes was maintained. Since the same function is used to generate the different surfaces and only the number of surface features differs, the $S_{a}$ value should be the same provided that enough features are present. Fig. (19b) clearly shows that this is the case. The $S_{a}$ value is oscillating around a constant value showing the corrugation of the surface down to a certain number of pixels defined by the size of the surface features in relation to the pixel size. At lower values of $\mathrm{N}, \log \left(\mathrm{S}_{\mathrm{a}}\right)$ is linearly related to $\log (\mathrm{N})$ with a slope close to two for these model surfaces, i.e. a fractal dimension close to three. The crossover between the constant and linear parts defines the smallest surface feature that can be detected. Consequently, the crossover point moves to $\operatorname{lower} \log (\mathrm{N})$ values as the number of surface features increases, i.e. gets smaller. In Fig. (19b) the data from the $\mathrm{MeX}^{\circledR}$ analysis are also plotted and the same general trend is observed. However, the crossover is larger compared with the power law analysis. In the power law analysis used in the present work the smallest area is located in the centre of the image and has the form of a cone. The linear part of the log-log plot corresponds to diminishing height of the cone and the crossover is the upper size of the cone. The fractal dimension is close to 3 since the volume of the cone is analysed at the smallest length scale. In the $\mathrm{MeX}^{\circledR}$ analyses the filtering smears out the surface features somewhat and the crossover is larger than expected theoretically. This is the same behaviour as observed for the experimental surfaces and shows the limitation of the $\mathrm{MeX}^{\circledR}$ analyses. A better resolution of the fine structure can be obtained by variable length analyses as shown for the model surfaces. However, in order to get better statistical values the surface roughness parameters should be calculated over the entire surface using different sizes of the analysing area in a similar way as described in Chauvy et al. [34]. This approach will be explored in a forthcoming paper.

\subsection{Comparison with In Vivo Experiments}

The main goal for characterising implant surfaces in great detail is to obtain a relationship between surface roughness parameters and the performance of the implants in vivo. Such a relationship would be beneficial for the development of new surfaces and would also minimize the need for animal studies. The relationship between in vivo results and surface roughness parameters such as $S_{a}$ has been previously emphasised [16, 32, 50, 51] but as described in Section 5.3 for example the fluid retention index seems to give contradictory correlation results. If we assume that the volume parameter used to calculate the fluid retention index is a better predictor of the in vivo performance then a correlation between this parameter and for example the removal torque (RTQ) value should exist. However, since volume parameters are very rarely given in the literature such a relationship will be difficult to prove. Since the volume parameters show the same general behaviour with filter size as the $S_{a}$ parameter, compare Figs. (5) and (18), the $S_{a}$ value can be used instead. Literature values from in vivo tests performed in rabbit, were collected for implant surfaces similar to the surfaces studied in the present paper [12, 5254]. In Fig. (20), removal torque data are plotted as a function of $S_{a}$. The data are collected from different sources and it may be questioned if a comparison is at all feasible. However, Fig. (20) clearly shows a linear relationship between removal torque and the surface roughness parameter $\mathrm{S}_{\mathrm{a}}$, with increasing removal torque values for surfaces with 
(a)
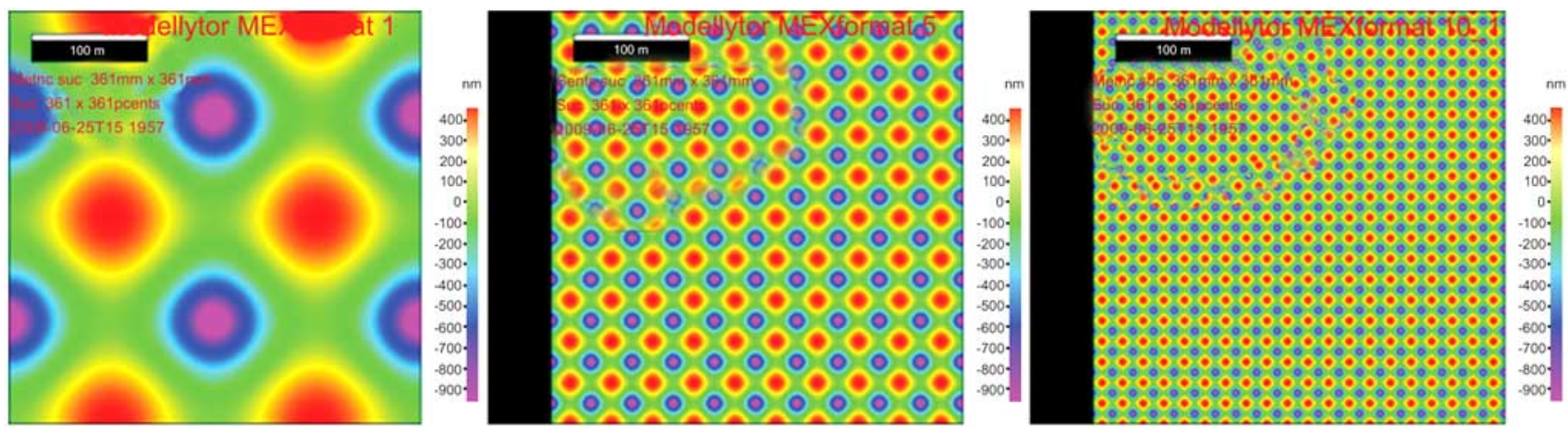

(b)

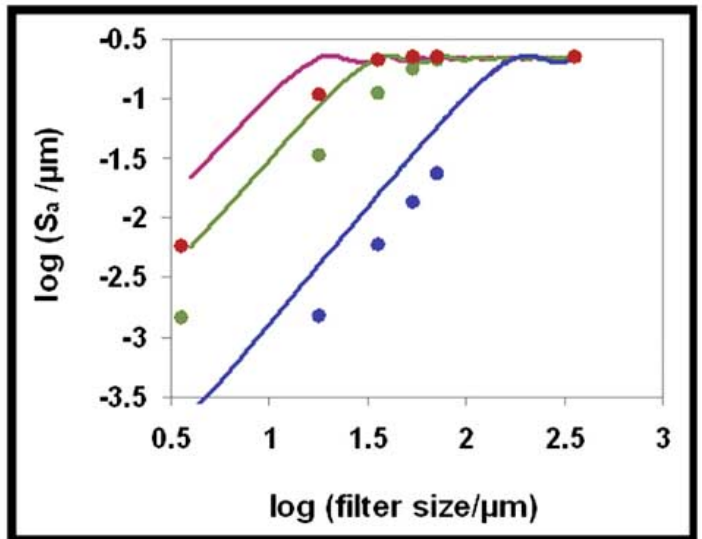

Fig. (19). (a) Model surfaces with 4, 100 and 400 protruding surface features, respectively, and $(\mathbf{b}) \log \left(\mathrm{S}_{\mathrm{a}}\right)$ as a function of $\log (\mathrm{N})$. The symbols are from the $\mathrm{MeX}^{\circledR}$ analysis and the lines from the power law analysis.

higher $S_{a}$ value. This applies both to data obtained after 1 and 3 months. Extrapolation to $S_{a}=0$, i.e. a surface without roughness, gives the same removal torque value for both 1 and 3 months test. This fact was used to get the relationship between removal torque and $S_{a}$ for the few data found for 12 months test.

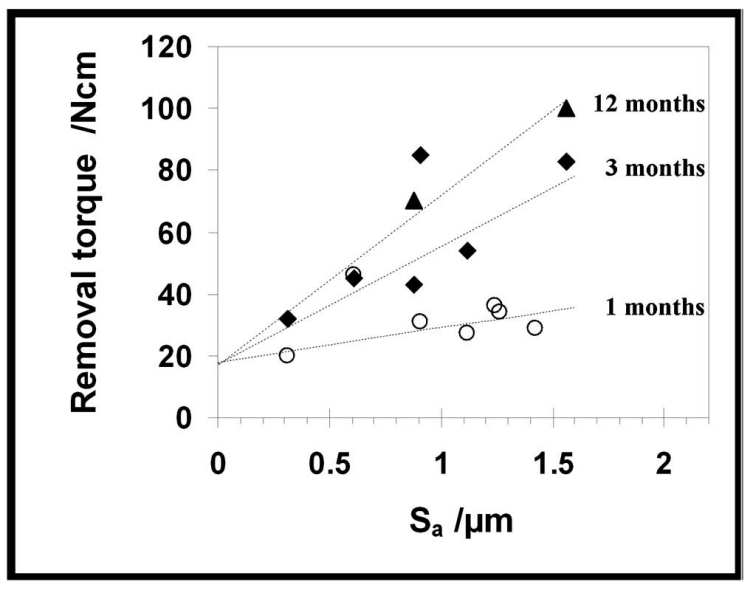

Fig. (20). Removal torque values on rabbit after one, three and 12 months. The surfaces studied were TS [12], FB [12, 52, 53], FB + HF [53], CB [54] and CB + HF [52, 54].

The removal torque data obtained after one month falls on a line and the HF treatment has minor additional effect on the removal torque after a short implantation time. One value for the FB surface falls outside the linear range [12] showing the same removal torque value after 3 weeks and 3 months. Also after 3 months, RTQ increases with increasing $S_{a}$ value for all surfaces except the fine blasted surface with $\mathrm{HF}$ etching [53], which shows a much higher removal torque value than expected. The reason for the high value is not known. However, in one study comparing the RTQ between coarse blasted surfaces with and without HF treatment increased RTQ was indeed found for the HF treated surface [54]. In addition, Ellingsen et al. [55] have shown improved bone-to-implant attachment for coarse blasted surfaces treated with hydrofluoric acid using pull-out experiments and in a recent study the semiconducting properties of the oxide were related to the enhanced performance [41]. Note that the data reported in Fig. (20) are restricted to turned and blasted surfaces with and without chemical etching with HF. Thus, for this restricted set of surfaces there is a clear relationship between the $S_{a}$ parameter and the removal torque value. This implies that ways of increasing $S_{a}$ for blasted surfaces, such as chemical etching and deposition, will give better anchoring results in vivo.

In order to look for some generality in the correlation between $S_{a}$ and removal torque other surfaces were also analysed. For aluminium oxide blasted surfaces [56] the removal torque values are lower compared with the $\mathrm{TiO}_{2}$ blasted surfaces but the general trend is the same, i.e. the removal torque value increases with increasing $S_{a}$ value. The 
presence of Bonit ${ }^{\circledR}$ (calcium phosphate) on the surface seems not to further increase the removal torque value indicating that the geometrical changes are dominating the in vivo performance. On the other hand, in the presence of hydroxyl apatite (HA) on fine blasted surfaces (FB) [12] the removal torque value after 3 and 12 weeks are much higher than expected from the relationship shown in Fig. (20).

A set of anodized surfaces have been subjected to an in vivo study measuring the removal torques values after 6 weeks [57]. Anodising to different potential results in a thicker titanium oxide layer but the $S_{a}$ value is only slightly increased. The removal torque values are fairly constant and little additional effect of the anodisation is observed.

In addition to the increase in surface roughness, there might also be a chemical stimulation as observed for hydroxyl apatite [12] and HF etching [53, 54]. Another example is inclusion of magnesium. In most cases the inclusion of $\mathrm{Mg}$ by oxidation of the titanium surface results in significantly higher removal torque values compared with changes in the surface roughness [12, 58, 59]. This illustrates that by a general comparison between surface roughness and in vivo results it is also possible to discriminate between a purely geometrical effect, a chemical effect, or a combination of both for the improved in vivo performance.

\section{CONCLUSIONS}

- $\quad$ AFM and 3D-SEM complement each other and by using a variable scale analysis the surface roughness at different levels can be obtained. 3D-SEM gives a good description of the roughness induced by physical treatment such as blasting while it is necessary to use AFM to reveal the surface roughness introduced by chemical etching.

- The filtering process used to study the scale dependence of the surface roughness parameters smears out the surface features somewhat and an apparent larger dimension is obtained but can be used for comparative studies. It is suggested that the present method should be complemented with calculations on unfiltered samples using a variable area approach.

- $\quad$ Out of the 13 parameters evaluated it was possible to discriminate between the $\mathrm{CB}, \mathrm{FB}$ and TS surfaces with 10 using the 3D-SEM technique. By the AFM technique the TS and TS+HF surfaces were well separated in 10 of the evaluated parameters. A separation of these surfaces with the 3D-SEM technique was only possible using the hybrid parameters $S_{\mathrm{dq}}$ and $\mathrm{S}_{\mathrm{dr}}$.

- $\quad$ The choice of parameters to be evaluated depends on the question asked. For correlation with in vivo measurements we suggest that besides the average height parameter, $S_{a}$, also the root-mean slope of the surface, $\mathrm{S}_{\mathrm{dq}}$, and the void volume, $\mathrm{V}_{\mathrm{vc}}$, should be evaluated.

- The surfaces studied all show fractal behaviour in certain length intervals. The fractal dimension was found to be higher for the blasted samples than for the turned sample. By measuring the crossover size it was also possible to discriminate between the coarse and fine blasted surfaces. Chemical etching with HF of a turned surface gives the same fractal dimension as for fine blasted surfaces but the crossover size is much lower showing the different level of roughness. The fractal dimension combined with the crossover size is a good complement to the scale dependent surface roughness parameters and should be determined when applicable.

- $\quad$ This work has stimulated the development of a local model for calculating the interface shear strength using the mean slope $\left(\mathrm{S}_{\mathrm{sl}}\right.$ in $\left.2 \mathrm{D}\right)$ and the root-meansquare slope $\left(S_{\mathrm{dq}}\right.$ in $\left.3 \mathrm{D}\right)$ of the surface [37].

\section{ACKNOWLEDGEMENTS}

Financial support from the Swedish Research Council (2005-21028-35344-27) is gratefully acknowledged. Alicona Imaging $\mathrm{GmbH}$ for support regarding $\mathrm{MeX}^{\circledR}$ issues.

\section{REFERENCES}

[1] Cooper LF, Zhou Y, Takebe J, et al. Fluoride modification effects on osteoblast behavior and bone formation at $\mathrm{TiO}_{2}$ grit-blasted c.p. titanium endosseous implants. Biomaterials 2006; 27: 926.

[2] Ellingsen J-E. Pre-treatment of titanium implants with fluoride improves their retention in bone. J Mater Sci Mat In Med 1995; 6: 749.

[3] Lamolle SF, Monjo M, Lyngstadaas SP, Ellingsen JE, Haugen HJ Titanium implant surface modification by cathodic reduction in hydrofluoric acid: surface characterization and in vivo performance. $\mathrm{J}$ Biomed Mater Res Part A 2009; 88A: 581.

[4] Lossdoerfer S, Schwartz Z, Wang L, et al. Microrough implant surface topographies increase osteogenesis by reducing osteoclast formation and activity. J Biomed Mater Res Part A 2004; 70A: 361

[5] Mustafa K, Wroblewski J, Hultenby K, Lopez BS, Arvidson K. Effects of titanium surfaces blasted with $\mathrm{TiO}_{2}$ particles on the initial attachment of cells derived from human mandibular bone. A scanning electron microscopic and histomorphometric analysis. Clin Oral Implants Res 2000;11:116.

[6] Nayab SN, Jones FH, Olsen I. Effects of calcium ion implantation on human bone cell interaction with titanium. Biomaterials 2005; 26: 4717.

[7] Brunette DM, Kzakbaznejad A, Takekawa M, et al. Improving the bioimplant interface by controlling cell behaviour using surface topography. In: Ellingsen JE, Lyngstadaas SP, editors. Bio-implant interface. Boca Raton: CRC Press, 2003. p.103.

[8] Brunski JB, Puleo DA, Nanci A. Biomaterials and Biomechanics of Oral and Maxillofacial Implants: Current Status and Future Developments. Int J Oral Maxillofac Impl 2000;15:15.

[9] Albrektsson T, Wennerberg A. Oral implant surfaces: Part 1--review focusing on topographic and chemical properties of different surfaces and in vivo responses to them. Int J Prosthodont 2004;17:536.

[10] Albrektsson T, Albrektsson B. Osseointegration of bone implants. A review of an alternative mode of fixation. Acta Orthop Scand 1987; 58: 567.

[11] Gotfredsen K, Nimb L, Hjorting-Hansen E, Jensen JS, Holmen A. Histomorphometric and removal torque analysis for $\mathrm{TiO}_{2}$-blasted titanium implants. An experimental study on dogs. Clin Oral Implants Res 1992; 3: 77.

[12] Gotfredsen K, Wennerberg A, Johansson C, Skovgaard IT, HjoertingHansen E. Anchorage of $\mathrm{TiO}_{2}$-blasted, HA-coated, and machined implants: an experimental study with rabbits. J Biomed Mater Res $1995 ; 29: 1223$.

[13] Wennerberg A. On Surface Roughness and Implant Incorporation Department of Biomaterials/Handicap Research. Göteborg: Göteborg University, 1996. p.125.

[14] Ericsson I, Johansson CB, Bystedt H, Norton MR. A histomorphometric evaluation of bone-to-implant contact on machineprepared and roughened titanium dental implants. A pilot study in the dog. Clin Oral Implants Res 1994; 5: 202.

[15] Ronold HJ, Ellingsen JE. Effect of micro-roughness produced by $\mathrm{TiO}_{2}$ blasting-tensile testing of bone attachment by using coin-shaped implants. Biomaterials 2002; 23: 4211.

[16] Wennerberg A, Albrektsson T, Johansson C, Andersson B. Experimental study of turned and grit-blasted screw-shaped implants 
with special emphasis on effects of blasting material and surface topography. Biomaterials 1996;17:15.

[17] Haberstroh KM. Hope for the short-term use of nanorough metallic implant formulations in the clinical arena. Nanomedicine 2006;1:355.

[18] Webster TJ, Ejiofor JU. Increased osteoblast adhesion on nanophase metals: Ti, Ti6Al4V, and CoCrMo. Biomaterials 2004; 25: 4731.

[19] Wieland M. Experimental determination and quantitative evaluation of the surface composition and topography of medical implant surfaces and their influence on osteoblastic cell-surface interactions. Zürich: Swiss Federal Institute of Technology Zurich, 1999.

[20] Deyneka-Dupriez N, Kocdemir B, Herr U, Fecht H-J, Wilke H-J, Claes L. Interfacial shear strength of titanium implants in bone is significantly improved by surface topographies with high pit density and microroughness. J Biomed Mater Res Part B 2007; 82B:305.

[21] Meirelles L. On Nano Size Strucutres For Enhanced Early Bone Formation. Department of Prosthodontics/Dental Material Schience, Department of Biomaterials. Göteborg: Göteborg University, 2007. p.70.

[22] Wieland M, Hanggi P, Hotz W, Textor M, Keller BA, Spencer ND. Wavelength-dependent measurement and evaluation of surface topographies: application of a new concept of window roughness and surface transfer function. Wear 2000; 237: 231.

[23] Wieland M, Textor M, Spencer ND, Brunette DM. Wavelengthdependent roughness: a quantitative approach to characterizing the topography of rough titanium surfaces. Int J Oral Maxillofac Implants 2001; 16:163.

[24] Voros J, Wieland M, Ruiz-Taylor L, Textor M, Brunette DM. Characterization of titanium surfaces. Titanium in Medicine 2001:87.

[25] Stout KJ, Blunt L. Nanometres to micrometres: three-dimensional surface measurement in bio-engineering. Surf Coat Technol 1995; 71: 69.

[26] SO 4287 - Geometrical Product Specifications (GPS) - Surface texture: Profile method - Terms, definitions and surface texture parameters. International Organization for Standardization, 1997; p. 25.

[27] ISO/DIS 25178-2 - Geometrical product specifications (GPS) Surface texture: Areal - Part 2: Terms, definitions and surface texture parameters. International Organization for Standardization, $2009 ;$ p.42.

[28] Dong WP, Sullivan PJ, Stout KJ. Comprehensive study of parameters for characterizing three-dimensional surface topography IV: Parameters for characterizing spatial and hybrid properties. Wear 1994;178: 45.

[29] Dong WP, Sullivan PJ, Stout KJ. Comprehensive study of parameters for characterizing three-dimensional surface topography III: Parameters for characterizing amplitude and some functional properties. Wear 1994;178: 29.

[30] Stout KJ, Ed. Development of methods for the characterisation of roughness in three dimensions. London: Penton Press, 2000.

[31] Arvidsson A, Sater Bashar A, Wennerberg A. The role of functional parameters for topographical characterization of bone-anchored implants. Clin Implant Dent Relat Res 2006; 8: 70.

[32] Wennerberg A, Albrektsson T. Suggested guidelines for the topographic evaluation of implant surfaces. Int $\mathrm{J}$ Oral Maxillofac Implants 2000;15: 331 .

[33] MacDonald W, Campbell P, Fisher J, Wennerberg A. Variation in surface texture measurements. J Biomed Mater Res Part B 2004; 70B: 262.

[34] Chauvy PF, Madore C, Landolt D. Variable length scale analysis of surface topography: characterization of titanium surfaces for biomedical applications. Surf Coat Technol 1998; 110: 48.

[35] Hansson S. Surface roughness parameters as predictors of anchorage strength in bone: a critical analysis. J Biomech Eng 2000;33:1297.

[36] Hansson S, Hansson KN. The effect of limited lateral resolution in the measurement of implant surface roughness: A computer simulation. J Biomed Mater Res Part A 2005; 75A: 472.
[37] Hansson S, Löberg J, Mattisson I, Ahlberg E. Characterisation of titanium dental implants. II: Local biomechanical model. Open Biomat J 2010; 2:14

[38] MeX 5.0. Alicona Imaging Gmbh, Graz, Austria 2007.

[39] Ponz E, Ladaga JL, Bonetto RD. Measuring surface topography with scanning electron microscopy. Part 1. EZEImage. A program to obtain 3D surface data. Microsc Microanal 2006; 12: 170.

[40] Hansson S, Norton M. The relation between surface roughness and interfacial shear strength for bone-anchored implants. A mathematical model. J Biomech 1999; 32: 829.

[41] Petersson I, Löberg J, Fredriksson A, Ahlberg E. Semi-conducting properties of titanium dioxide surfaces on titanium implants. Biomaterials 2009; 30: 4471.

[42] Thomas TR, Rosen BG, Amini N. Fractal characterisation of the anisotropy of rough surfaces. Wear 1999; 232: 41.

[43] Zinger O, Chauvy PF, Landolt D. Scale-resolved electrochemica surface structuring of titanium for biological applications. J Electrochem Soc 2003; 150: B495.

[44] Burger J, Dietler G, Binggeli M, Christoph R, Marti O. Aspects of the surface roughness of ceramic bonding tools on a nanometer scale investigated with atomic force microscopy. Thin Solid Films 1994; 253 : 308.

[45] Brown CA, Charles PD, Johnsen WA, Chesters S. Fractal analysis of topographic data by the patchwork method. Wear 1993; 161: 61.

[46] Bakucz P, Krueger-Sehm R. A new wavelet filtering for analysis of fractal engineering surfaces. Wear 2009; 266: 539.

[47] Buzio R, Malyska K, Rymuza Z, et al. Experimental investigation of the contact mechanics of rough fractal surfaces. IEEE Trans Nanobiosci 2004; $3: 27$.

[48] Gagnepain JJ, Roques-Carmes C. Fractal approach to two-dimensional and three-dimensional surface roughness. Wear 1986;109:119.

[49] Russ JC. Fractal Surfaces. New York: Plenum Press, 1994.

[50] Wennerberg A, Albrektsson T, Lausmaa J. Torque and histomorphometric evaluation of c.p. titanium screws blasted with 25- and 75-mmsized particles of Al2O3. J Biomed Mater Res 1996; 30: 251.

[51] Shalabi MM, Gortemaker A, Van't Hof MA, Jansen JA, Creugers NHJ. Implant surface roughness and bone healing: a systematic review. J Dent Res 2006; 85: 496.

[52] Johansson CB, Wennerberg A, Boström-Junemo K, Holmen A, Hansson $\mathrm{S}$. In vivo comparison of $\mathrm{TiO}_{2}$ blasted- and fluoride modified implants in rabbit bone. 7th World Biomaterials Congress. Sydney, 2004.

[53] Ellingsen J-E, Johansson C, Wennerberg A, Holmen A. Improved retention and bone-to-implant contact with fluoride-modified titanium implants. Int J Oral Maxillofac Implants 2004;19: 659.

[54] Meirelles L, Currie F, Jacobsson M, Albrektsson T, Wennerberg A. The effect of chemical and nanotopographical modifications on the early stages of osseointegration. Int J Oral Maxillofac Implants 2008; 23: 641.

[55] Ellingsen JE, Ronold HJ, Boström KJ, Holmen A, Hansson S Enhanced bone-to-implant attachment of fluoride-modified titanium implants. IADR. Gothenburg, 2003.

[56] Reigstad O, Franke-Stenport V, Johansson CB, Wennerberg A, Roekkum M, Reigstad A. Improved bone ingrowth and fixation with a thin calcium phosphate coating intended for complete resorption. J Biomed Mater Res Part B 2007; 83B:9.

[57] Sul Y-T, Johansson Carina B, Jeong Y, Wennerberg A, Albrektsson T. Resonance frequency and removal torque analysis of implants with turned and anodized surface oxides. Clin Oral Implants Res 2002; 13: 252.

[58] Sul Y-T, Johansson C, Byon E, Albrektsson T. The bone response of oxidized bioactive and non-bioactive titanium implants. Biomaterials 2005; 26: 6720 .

[59] Sul Y-T, Johansson C, Albrektsson T. Which surface properties enhance bone response to implants? Comparison of oxidized magnesium, TiUnite, and Osseotite implant surfaces. Int J Prosthodont 2006; 19: 319 . 